\title{
Photo absorption enhancement in strained silicon nanowires: An atomistic study
}

\author{
Daryoush Shiri, ${ }^{1, a)}$ M. Golam Rabbani, ${ }^{2, b)}$ Jianqing Qi, ${ }^{2}$ Andrei K. Buin, ${ }^{3, c)}$ \\ and M. P. Anantram ${ }^{2}$ \\ ${ }^{1}$ Institute for Quantum Computing (IQC), Department of Physics and Astronomy, University of Waterloo, \\ Waterloo, Ontario N2L 3G1, Canada \\ ${ }^{2}$ Electrical Engineering Department, University of Washington, Seattle, Washington 98195-2500, USA \\ ${ }^{3}$ Department of Electrical Engineering, University of Toronto, Toronto, Ontario M5S 2J7, Canada
}

(Received 7 February 2017; accepted 29 June 2017; published online 17 July 2017)

\begin{abstract}
The absorption spectra of silicon nanowires are calculated using semi-empirical $\mathrm{sp}^{3} \mathrm{~d}^{5} \mathrm{~s}^{*}$ tight binding and Density Functional Theory methods. The roles of diameter, wave function symmetry, strain, and crystallographic direction in determining the absorption are discussed. We find that compressive strain can change the band edge absorption by more than one order of magnitude due to the change in wave function symmetry. In addition, photon polarization with respect to the nanowire axis significantly alters the band edge absorption. Overall, the band edge absorption of [110] and [100] silicon nanowires can differ by as much as three orders of magnitude. We find that compared to bulk Silicon, a strained Silicon nanowire array can absorb infrared photons $(1.1 \mathrm{eV})$ approximately one hundred times better. Finally, we compare a fully numerical and a computationally efficient semi-analytical method, and find that they both yield satisfactory values of the band edge absorption. Published by AIP Publishing. [http://dx.doi.org/10.1063/1.4993587]
\end{abstract}

\section{INTRODUCTION}

Silicon nanowires (SiNWs) have been actively studied both experimentally and theoretically. Compatibility with traditional silicon microelectronic fabrication methods, and demonstrations of quantum confinement, tunable bandgap, and sensitivity of the electronic structure to surface adsorbents and mechanical strain offer significant long-term prospects in applications. During the last decade, we have witnessed applications in transistors, ${ }^{1,2}$ logic circuits, ${ }^{3}$ memory, ${ }^{4}$ spin-based quantum computing, ${ }^{5}$ chemical, ${ }^{6}$ and biological ${ }^{7}$ sensors, piezo-resistive sensors, ${ }^{8}$ nano mechanical resonators, ${ }^{9}$ and thermoelectric converters. ${ }^{10,11}$ The utilization of SiNWs in optoelectronic devices such as solar cells, ${ }^{12,13}$ photo-transistors, ${ }^{14,15}$ and avalanche photodiodes ${ }^{16,17}$ is also promising. Integration of silicon with photonics is important due to low cost and mature fabrication technology of silicon chips. Over the years, researchers have attempted to observe light emission with the signature of quantum confinement from SiNWs. The availability of new fabrication methods ${ }^{18}$ has made it possible to reduce non-radiative and surface recombination rates. This is important for light emission arising from transition between conduction and valence band states (as opposed to defect states). At a more fundamental level, light emission/absorption at bandgap energies in SiNWs mandates having a direct bandgap and a symmetry allowed dipole transition between states at the conduction (c) and valence (v) band edges,

\footnotetext{
a) Author to whom correspondence should be addressed: daryoush.shiri@ chalmers.se. Present address: Department of Physics, Chalmers University of Technology, SE 41296 Göteborg, Sweden.

${ }^{b)}$ Present address: Intel, Inc., Portland, Oregon 97124, USA.

${ }^{c}$ Present address: D\&D Integrative Care, Inc., Toronto, Ontario M5J 2H7, Canada.
}

$$
\left\langle\Psi_{c}(\boldsymbol{r})|\boldsymbol{r}| \Psi_{v}(\boldsymbol{r})\right\rangle=\int \Psi_{c}^{*}(\boldsymbol{r}) \boldsymbol{r} \Psi_{v}(\boldsymbol{r}) d^{3} \boldsymbol{r} .
$$

To have a non-zero (symmetry allowed) value for the dipole moment, the integrand in Eq. (1) should be of even symmetry. Here, $\Psi_{\mathrm{c}}$ and $\Psi_{\mathrm{v}}$ are the conduction and valence band wave functions, and $\mathbf{r}$ is the position operator. In bulk silicon and large diameter SiNWs, the conduction band minimum and valence band maximum do not have the same wave vector value within the Brillouin Zone (BZ) [Fig. 1(a)]. Since the photon absorption/emission is a momentum conserving process, indirect transitions are weak second order processes mediated by phonons. However, narrow diameter SiNWs can have a direct bandgap due to folding of off center energy states of bulk silicon into the BZ center. Figure 1(b) shows a direct band gap for a [100] SiNW with a diameter of $2.2 \mathrm{~nm}$. However, most experiments have involved large diameter SiNWs within a hybrid light emitting device, where photon emission occurs efficiently via a direct bandgap nanowire made from III-V materials. In these demonstrations, the SiNW is used only for biasing and carrier injection. For example, nano-lasers and Light Emitting Diodes (LEDs) were reported based on the combination of top-down grown SiNWs and bottom-up III- $\mathrm{V}^{19}$ and Cd-VI nanowires, ${ }^{20}$ as well as a core/shell $\mathrm{p}$-Si/n-CdS nanowire. ${ }^{17}$ Further, studies utilizing SiNW arrays in solar cells, wave guides,${ }^{21}$ color filters, ${ }^{22}$ and photonic crystals exploit the electromagnetic wave scattering properties of these arrays rather than the quantum confined properties of SiNWs because their diameters are between few tens of nanometers and a $1 \mu \mathrm{m}$. The role of SiNW arrays in these experiments is to help with (a) Spectrum Widening and (b) Collimation and Lensing. Spectrum Widening refers to the increase in optical absorption of a SiNW array over a wider wavelength range than thin film silicon of equivalent 

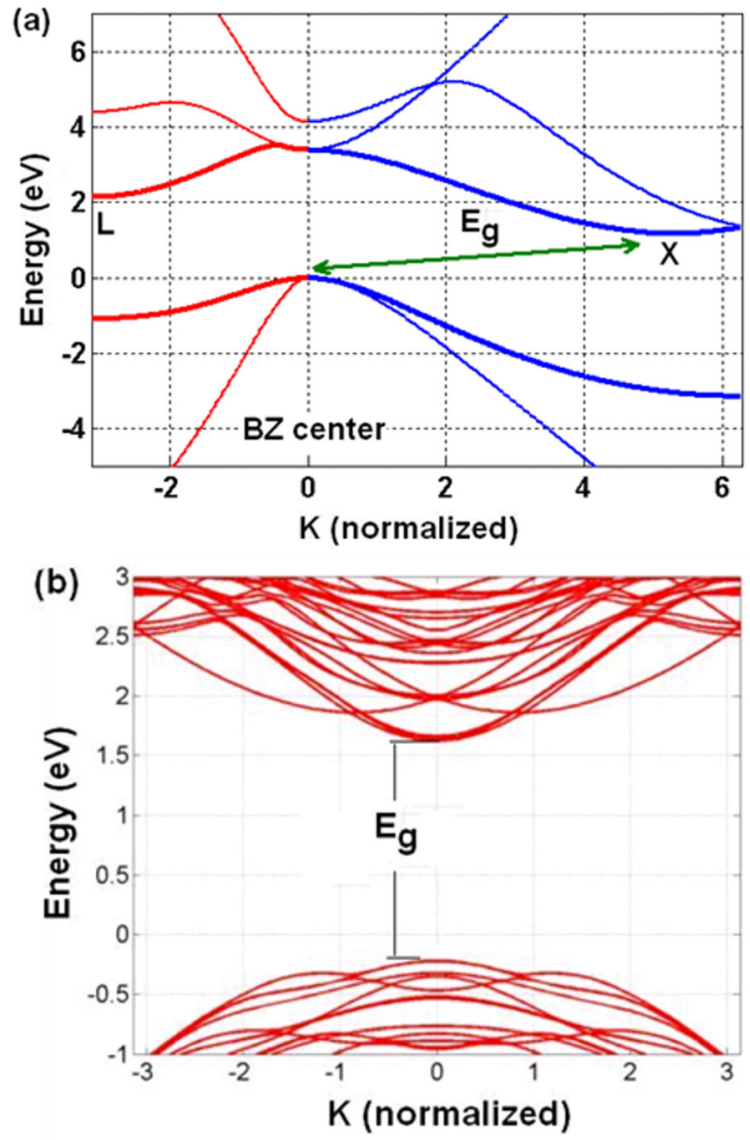

FIG. 1. (a) Band structure of bulk silicon showing an indirect bandgap. (b) Band structure of a [100] SiNW with a diameter of $2.2 \mathrm{~nm}$ shows a direct bandgap in contrast to bulk silicon.

thickness. ${ }^{23-26}$ Collimation and lensing refers to the ability of a periodic nanowire array to collimate $\operatorname{IR}(\lambda=1500 \mathrm{~nm})$ wavelengths to increase absorption. ${ }^{27,28}$ Finally, there are examples of prototype devices using a single large diameter SiNW as photodiodes, solar cells, and photo transistors. A detailed review of these experiments and the performance of devices can be found elsewhere. ${ }^{29}$

The role of strain in engineering electronic and optoelectronic properties has proved important in bulk materials (e.g., $\mathrm{Si}, \mathrm{Ge}$ ). The residual stress due to lattice mismatch between silicon and germanium can enhance the carrier mobility in Metal-Oxide-Semiconductor (MOS) transistors. Additionally, it has been demonstrated that growing a germanium layer on a silicon substrate can decrease the splitting between the indirect and direct bandgap in germanium. ${ }^{30,31}$ The reduction in splitting in turn increases the light emission efficiency of optically $^{32}$ and electrically pumped ${ }^{33} \mathrm{Ge}$-on-Si. On the computational side, using the tight binding (TB) method, it was shown that $\mathrm{a}+2 \%$ strain value changes the bandgap of bulk Ge from indirect to direct. ${ }^{34}$ This value of strain is similar to the value of $+1.8 \%$ predicted by the effective mass theory ${ }^{31}$ as well as the experimental value of $+2 \%$ reported in Ge-on-Si light emitting diodes. ${ }^{32,33}$ The value of strain in these thin films is fixed because it is controlled by alloy composition. ${ }^{35,36}$ Similarly, coaxial SiNWs can be strained by a lattice mismatch induced by the shell material; examples include germanium, ${ }^{37} \mathrm{SiO}_{2},{ }^{18,38-41}$ silica $^{42}$ metallization, ${ }^{2}$ and silicon nitride. $^{43,44}$ Strain was also found to modulate the spontaneous emission rate in narrow diameter SiNWs by two orders of magnitude ${ }^{45}$ as a result of symmetry change in wave functions and a direct to indirect bandgap transition. ${ }^{46-53}$ It was recently observed that the electron-hole recombination rate in silicon nanowires can be enhanced by 6 times due to strain $(1 \%-5 \%$ tensile) induced in the bent sections of the nanowires. ${ }^{54}$

Motivated by the effect of strain on modulating the spontaneous emission rate, the main goal of this article is the computational study of photo absorption in SiNWs and its dependence on strain, diameter, and crystallographic direction. Due to the equivalence of the stimulated emission and absorption rates $\left(\mathrm{R}_{\mathrm{stim}} \sim \mathrm{R}_{\mathrm{abs}}\right)$, this study facilitates the calculation of the gain spectrum in SiNW-based lasers as well. We start from the quantum mechanical formulation of the dielectric function of semiconductors adapted for a nanowire by including 1D Joint Density of States (JDOS) using the ten orbital $\left(\mathrm{sp}^{3} \mathrm{~d}^{5} \mathrm{~s}^{*}\right)$ semi-empirical tight binding method, ${ }^{55} \mathrm{We}$ note that bandgap underestimation in Density Functional Theory (DFT) makes direct comparison of light absorption in SiNWs with bulk silicon less straightforward. On the other hand, TB has already shown success in reproducing the band structure of bulk silicon as well as the experimental strain-induced shift of photoluminescence (PL) spectra in SiNWs. ${ }^{18,56}$

The absorption spectrum can be found using both real and imaginary parts of the dielectric function. The real part of the dielectric function is calculated from the imaginary part using the Kramers-Krönig relationship. The approximations can be summarized as follows: (a) the tight binding method is used, and is validated by comparing with $\mathrm{DFT}^{57}$ and experiments in case of bulk Silicon. (b) The optical transitions studied in this work are all of direct type (first order) and indirect (second order phonon mediated) transitions are not studied here. (c) After observing close agreement of the full band (fully numerical) and effective mass (semi analytic) approximations, the latter is chosen. The transition matrix element value is assumed to be close to the value at the Brillouin zone center. (d) The local field effect and many-body effect are not considered in this work. (e) The calculations are carried out only to the linear response. (f) In evaluating the matrix elements, it is assumed that the orbitals of Silicon and Hydrogen are of Slater type. In carrying out the validation mentioned in (a), we use the DFT method implemented in SIESTA ${ }^{57}$ to calculate the absorption spectrum of bulk silicon only due to direct transitions. Calculation of the absorption in indirect bandgap semiconductors has been discussed in the literature ${ }^{58}$ and a recent DFT study reports the calculation of the absorption spectrum (phonon assisted part) in bulk silicon using the second order perturbation theory. ${ }^{59}$

This article is organized as follows. We start with a review of computational methods, i.e., energy minimization with and without strain, and band structure calculations using the tight binding method (Sec. II A). This is followed by a comparison of a fully numerical and a computationally efficient semi-analytical computation of the absorption spectrum using the TB method (Sec. II B 1). The results from these computations are compared to DFT-based and experimental 
absorption spectra for bulk silicon (Sec. II B 2). Then, the effects of diameter, crystallographic direction, and strain value on band edge absorption of SiNWs are discussed along with an explanation of the underlying principles (Sec. III). Section IV concludes the article.

\section{METHODS}

\section{A. Energy minimization and strain application}

The SIESTA package ${ }^{57}$ is used to relax the atomic structure of periodic silicon nanowires. ${ }^{60}$ The Local Density Approximation (LDA) functional with Perdew-Wang (PW91) exchange correlation potential ${ }^{61}$ is used, along with spin polarized Kohn-Sham orbitals of double- $\xi$ type with polarization. The Brillion Zone is sampled by a set of $1 \times 1 \times 40 \mathrm{k}$ points along the nanowire axis ( $\mathrm{z}$ axis). The minimum center to center distance of SiNWs is at least $6 \mathrm{~nm}$ to avoid inter-cell interaction. The values for energy cut-off, split norm, and force tolerance are $680 \mathrm{eV}(50 \mathrm{Ry}), 0.15$, and $0.01 \mathrm{eV} / \AA$, respectively. The energy of the unstrained nanowire is minimized using the Conjugate Gradient (CG) algorithm during which the variable unit cell option is used. An initial guess for the strained nanowire is generated by uniformly stretching the unstrained nanowire according to $\mathrm{a}_{\text {new }}=\mathrm{a}_{\text {old }}(1 \pm \varepsilon)$, where a is the unit cell length and $\varepsilon$ is the strain value in percent [see Fig. 2(a)]. At nonzero values of strain, the unit cell is relaxed using the constant volume (fixed unit cell) option.

We calculate the band structure and Eigen states using the ten orbital $\left(\mathrm{sp}^{3} \mathrm{~d}^{5} \mathrm{~s}^{*}\right)$ TB method with Jancu's parameters. ${ }^{55}$ The bandgap underestimation that is typical of DFT calculations is avoided by using the TB method. The TB method has successfully reproduced the effect of radial strain on the PL spectrum of narrow SiNWs in the experiments. ${ }^{18,56}$ The trend of the TB bandgap change with diameter in the case of silicon nano-crystals also agrees with DFT based calculations. ${ }^{62}$ In the case of [110] SiNWs, the TB method reproduces the bandgap change with diameter observed in Scanning Tunneling Spectroscopy (STS) measurements. ${ }^{63}$ Finally, we note that the nanowires in this work are constructed from a bulk silicon crystal in [110] and [100] directions terminating their surface with hydrogen atoms. The cross section of each nanowire lies in the $x-y$ plane and its axis is parallel to the $\mathrm{z}$ direction. Figure 2(a) shows the atomic structure of a unit cell in a SiNW oriented in the [110] direction with a diameter of $1.7 \mathrm{~nm}$.

\section{B. Photo absorption}

The calculation of the tensor dielectric function $\left(\epsilon_{\alpha \beta}\right)$ of the material is required to obtain the absorption spectrum. Macroscopically, the displacement vector (D) and applied electric field $(\mathbf{E})$ are related by

$$
\boldsymbol{D}_{\alpha}(\boldsymbol{q}, \omega)=\epsilon_{\alpha \beta}(\boldsymbol{q}, \omega) \boldsymbol{E}_{\beta}(\boldsymbol{q}, \omega) .
$$

$\omega$ and $\boldsymbol{q}$ are frequency and wave vector of the electromagnetic field. For anisotropic solids (non-cubic crystals), the non-diagonal elements of $\varepsilon_{\alpha \beta}$ are nonzero. In addition to the above constitutive equation, the electric field induces a current density $(J)$ in the material, which is given by
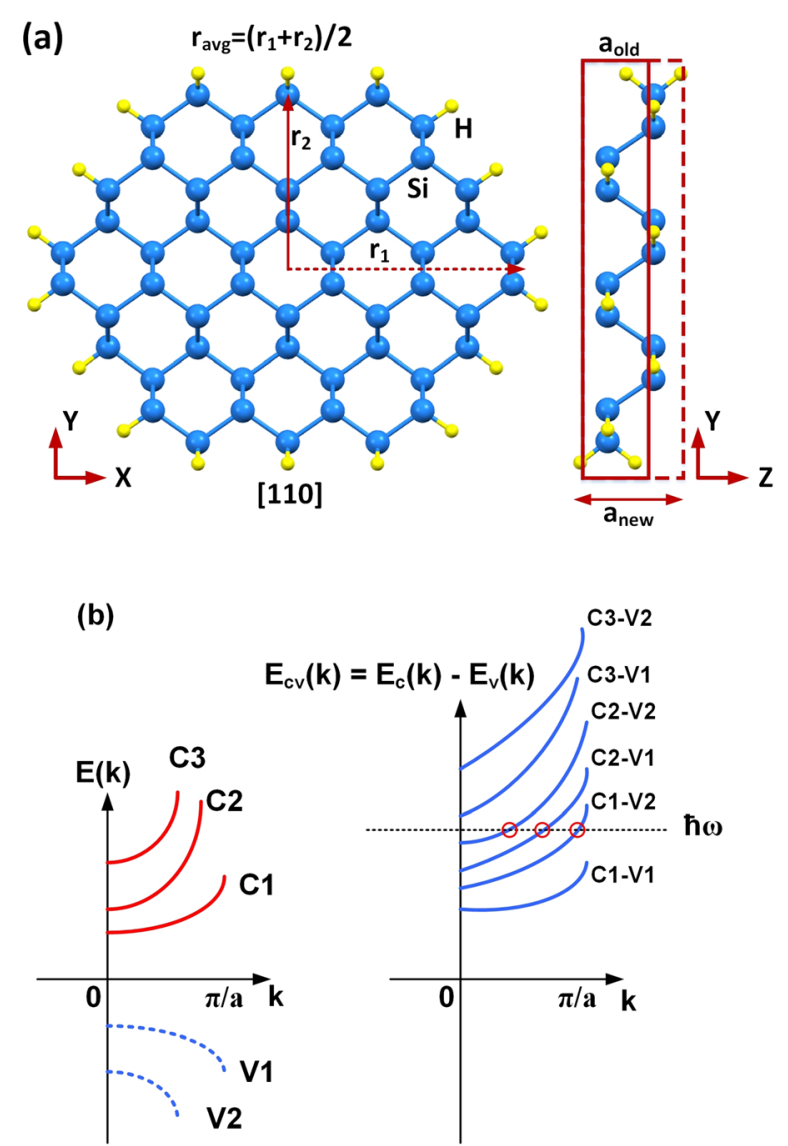

FIG. 2. (a) Illustration of a [110] SiNW unit cell with a diameter of $1.7 \mathrm{~nm}$. The diameter is defined as the average of large and small diameters. Dark and bright atoms correspond to $\mathrm{Si}$ and $\mathrm{H}$, respectively. Application of strain is performed by updating the old unit cell $\left(\mathrm{a}_{\text {old }}\right)$ by the given strain percent (c). (b) Pictorial representation of finding/counting the number of available inter-band optical transitions between valence (v) and conduction (c) bands for a given photon energy $\hbar \omega$.

$$
J_{\alpha(}(\boldsymbol{q}, \omega)=-\frac{i \omega}{4 \pi}\left[\epsilon_{\alpha \beta}(\boldsymbol{q}, \omega)-\delta_{\alpha \beta}\right] E_{\beta}(\boldsymbol{q}, \omega) .
$$

$\delta_{\alpha \beta}$ is the Krönecker delta function. The quantum mechanical treatment to calculate the dielectric function of solids was initially demonstrated in Refs. 64-66. Later, this method was used for calculation of optical properties of bulk copper ${ }^{67}$ and silicon nano crystals. ${ }^{68}$ The procedure is summarized here. The single particle Hamiltonian of the periodic solid with the time-dependent magnetic $(\mathbf{A}(\mathbf{r}, \mathbf{t}))$ and electric $(\mathrm{V}(\mathrm{r}, \mathrm{t}))$ potentials is

$$
\mathcal{H}=\frac{1}{2 m}\left[\mathbf{p}+\left(\frac{e \mathbf{A}(\mathbf{r}, \mathrm{t})}{c}\right)\right]^{2}+V(\boldsymbol{r}, t)+U(\boldsymbol{r}) .
$$

The unperturbed periodic potential in the crystal is $\mathrm{U}(\mathbf{r})$. Since the laser power used in the photoluminescence (PL) studies of SiNWs is typically in the $\mathrm{kW} / \mathrm{cm}^{2}$ range, it is possible to ignore the nonlinear perturbation terms $\left(\mathrm{A}^{2}\right)$, and retain only the linear terms. The response of electrons in the solid to the perturbation results in a change in charge $(\rho(r, t))$ and current densities. The charge and current densities are in turn related by the continuity equation, $\nabla . J+\partial \rho / \partial t=0$. The change in charge and current densities from equilibrium can 
be obtained from knowledge of the density matrix $(\wp)$, which is obtained by solving the Liouville equation,

$$
i \hbar \frac{\partial \wp}{\partial t}=[\mathcal{H}, \wp] .
$$

The density matrix can be written as, $\wp=\wp^{0}+\wp^{1}$, where the first and the second terms correspond to the unperturbed and perturbed density matrix, respectively. The unperturbed part is given by

$$
\wp^{0}|\boldsymbol{k} l\rangle=f_{0}\left(E_{k l}\right)|\boldsymbol{k} l\rangle,
$$

where $f_{0}$ is the Fermi function. $\mathbf{k}$ and $l$ are quantum numbers that represent the electron wave vector and band index of the unperturbed wave function,

$$
|\boldsymbol{k} l\rangle=\frac{1}{\sqrt{V}} e^{i k . r} u_{k l}
$$

with energy Eigen value $E_{k l} . \mathrm{V}$ is the volume of the crystal and $u_{k l}$ is the periodic part of the wave function. From the first order perturbed density matrix $\left(\wp^{1}\right)$, the induced charge density can be found using

$$
\rho^{\text {ind }}(\boldsymbol{r}, t)=\operatorname{Trace}\left(\wp^{1}\right) \rho_{\text {op }}^{0}(\boldsymbol{r}),
$$

$\rho_{o p}^{0}(\boldsymbol{r})$ is the quantum mechanical charge density operator, $\rho_{o p}^{0}(\boldsymbol{r})=e \delta\left(\boldsymbol{r}-\boldsymbol{r}_{\boldsymbol{e}}\right)$, where $e$ and $r_{e}$ represent the charge of the electron and its position in the crystal, respectively. Inserting $\wp$ into Eq. (5) and multiplying it from right (left) by $|\boldsymbol{k} l\rangle\left(\left\langle\boldsymbol{k}+\boldsymbol{q} l^{\prime}\right|\right)$ makes it possible to find $\wp^{1}$ and $\rho^{\text {ind }}(\boldsymbol{r}, t)$. The band index of the new state is shown by $l$ ' and wave vector change of the electron is due to absorption/emission of a photon with a wave vector of $\mathbf{q}$. Using $\rho^{\text {ind }}(\boldsymbol{r}, t)$ and continuity equation, the value of $J^{\text {ind }}(\boldsymbol{r}, t)$ is known at this stage. Finally, $\varepsilon(\mathbf{q}, \omega)$ can be calculated by using Eq. (3) from knowledge of $J^{\text {ind }}(\boldsymbol{r}, t)$. If it is assumed that the wavelength of the incoming light is larger than the unit cell length $\left(\mathrm{U}_{\mathrm{c}}\right)$ of the solid $\left(\lambda \gg U_{c}\right)$, then it can be said that $q=2 \pi / \lambda$ is zero and the dielectric tensor is given by

$$
\begin{aligned}
\varepsilon(0, \omega)=1 & -\frac{4 \pi e^{2} N}{m V \omega^{2}} \\
& +\frac{4 \pi e^{2}}{m^{2} V \omega^{2}} \sum_{l l^{\prime} k} \frac{\left|\left\langle l k|\boldsymbol{p}| l^{\prime} k\right\rangle\right|^{2}\left(f_{0}\left(E_{l^{\prime} k}\right)-f_{0}\left(E_{l k}\right)\right)}{\hbar \omega+E_{l^{\prime} k}-E_{l k}+i \eta} .
\end{aligned}
$$

Here, $\mathrm{N}$ is the number of unit cells in the crystal and $\eta$ is the broadening factor. From now on, we replace $l$ and $l^{\prime}$ with $\mathrm{v}$ and $\mathrm{c}$ which represent quantum numbers of a state in valence and conduction bands, respectively. In the process of obtaining Eq. (9), the Local Field Effect (LFE) was ignored, i.e., it was assumed that there is no distinction between macroscopic and microscopic potentials and electric fields. Otherwise a microscopic potential, $\mathrm{V}^{\mathrm{ind}}(\boldsymbol{r}, \mathrm{t})$, is induced from the macroscopic excitation which is $\mathrm{V}(\boldsymbol{r}, \mathrm{t})$. Hence before starting the previous procedure, e.g., Eq. (8), $\mathrm{V}^{\text {ind }}(\boldsymbol{r}, \mathrm{t})$ must be first calculated from $\mathrm{V}(\boldsymbol{r}, \mathrm{t})$ using perturbation theory. Later on, $\mathrm{V}^{\text {ind }}(\boldsymbol{r}, \mathrm{t})$ enters the main procedure as a new perturbation (as if LFE was not included).
In addition to the linear response, the following assumptions were made in our calculations: (a) Nanowire is assumed to be an isotropic media, i.e., $\mathbf{D}$ is always parallel to $\mathbf{E}$. This is because for cubic crystals like silicon the dielectric tensor is isotropic. (b) Although Eq. (9) is general and it includes both intra and inter sub band transitions, only the inter sub band transitions are considered for SiNWs [See Fig. 2(b)]. This is because the bandgap of SiNWs is large and at room temperature the conduction band is empty. However, in case of a small bandgap material, e.g., bismuth nanowire ${ }^{69}$ with $\mathrm{E}_{\mathrm{g}}=123 \mathrm{meV}$, the conduction band has a significant number of electrons and transitions between conduction band states $\left(\mathrm{C}_{1}\right.$ to $\mathrm{C}_{2}$ and $\mathrm{C}_{1}$ to $\mathrm{C}_{3}$ etc) cannot be ignored at room temperature [Fig. 2(b)]. (c) As a result of the previous assumption (b), the conduction band is empty and the valence band is full, i.e., $f_{0}\left(E_{v k}\right)=0$ and $f_{0}\left(E_{c k}\right)=1$ (This is equivalent to assuming $\mathrm{T}=0 \mathrm{~K}$.).

Finding the imaginary part of $\varepsilon(0, \omega)$ [Eq. (9)] is straightforward. Converting CGS to SI unit (multiplying to $1 / 4 \pi \varepsilon_{0}$ ) and using the Lorentzian approximation of Dirac's delta function [Eq. (10)]

$$
\delta\left(\hbar \omega+E_{c k}-E_{v k}\right)=\frac{1}{\pi} \frac{\eta}{\left(\hbar \omega+E_{c k}-E_{v k}\right)^{2}+\eta^{2}}
$$

results in Eq. $(11)^{70,71}$

$$
\begin{aligned}
\varepsilon_{2}(\omega) & =\operatorname{imag}(\varepsilon(0, \omega)) \\
& =\frac{\pi e^{2}}{\varepsilon_{0} m^{2} V \omega^{2}} \sum_{c v k}\left|\left\langle u_{v k}|\hat{\boldsymbol{e}} \cdot \boldsymbol{p}| u_{c k}\right\rangle\right|^{2} \delta\left(\hbar \omega+E_{c k}-E_{v k}\right) .
\end{aligned}
$$

$\hat{e}$ is a unit vector along the direction of photon polarization and $\varepsilon_{0}$ is dielectric permittivity of vacuum. Now the momentum matrix element between a conduction band state $\left(\mathrm{c}, \boldsymbol{k}^{\prime}\right)$ and a valence band state (v, $\boldsymbol{k})$ can be written as $\left\langle u_{v k}|\boldsymbol{p}| u_{c k^{\prime}}\right\rangle$ or $\left\langle v, \boldsymbol{k}|\boldsymbol{p}| c, \boldsymbol{k}^{\prime}\right\rangle$. Using the time evolution of the position operator, $\frac{\partial}{\partial t} \boldsymbol{r}=\frac{i}{\hbar}[H, \boldsymbol{r}],{ }^{58}$ the momentum matrix element can be written in terms of position operator as follows. The Hamiltonian operator acts on each state according to $H|n, \boldsymbol{k}\rangle$ $=E_{n, \boldsymbol{k}}|n, \boldsymbol{k}\rangle$ and therefore

$$
\begin{aligned}
\left\langle v, \boldsymbol{k}|\mathbf{p}| c, \boldsymbol{k}^{\prime}\right\rangle & =\left\langle v, \boldsymbol{k}\left|m_{0} \frac{\partial}{\partial t} \mathbf{r}\right| c, \boldsymbol{k}^{\prime}\right\rangle \\
& =\frac{i m_{0}}{\hbar}\left\langle v, \boldsymbol{k}|H \mathbf{r}-\mathbf{r} H| c, \boldsymbol{k}^{\prime}\right\rangle \\
& =\frac{i m_{0}}{\hbar}\left(E_{v, \boldsymbol{k}}-E_{c, \boldsymbol{k}^{\prime}}\right)\left\langle v, \boldsymbol{k}|\mathbf{r}| c, \boldsymbol{k}^{\prime}\right\rangle .
\end{aligned}
$$

Now, the momentum matrix element in Eq. (11) can be replaced by

$$
\left|\left\langle u_{v, k}|\boldsymbol{p}| u_{c, k^{\prime}}\right\rangle\right|^{2}=m^{2} \omega_{c v}{ }^{2}\left|\left\langle u_{v}|\mathbf{r}| u_{c}\right\rangle\right|^{2},
$$

where $\omega_{c v}=\left(E_{v, \boldsymbol{k}}-E_{c, \boldsymbol{k}^{\prime}}\right) / \hbar$.

To find the position matrix element in Eq. (13), we use Slater-type ${ }^{34}$ orbitals, which have been successfully applied to semiconductors ${ }^{72-75}$ and molecules. ${ }^{76}$ We start with an electronic Eigen-state given at a point along the 1D BZ of 
SiNW which is expanded using the 10 orbital basis. The number of Eigen values for each wave vector is $N_{\text {orbit }}=N_{S i}$ $\times 10+N_{H}$, where $\mathrm{N}_{\mathrm{Si}}$ and $\mathrm{N}_{\mathrm{H}}$ are the number of silicon and hydrogen atoms within each unit cell, respectively. The position matrix element in Eq. (13) can be reduced to two terms, ${ }^{34,72,76}$

$$
\begin{aligned}
\left\langle v, \boldsymbol{k}|\mathbf{r}| c, \boldsymbol{k}^{\prime}\right\rangle= & \sum_{\alpha \beta} C_{v \alpha}^{*}(\boldsymbol{k}) C_{c \beta}\left(\boldsymbol{k}^{\prime}\right) \delta_{\boldsymbol{k}, \boldsymbol{k}^{\prime}}\langle\alpha(\boldsymbol{r})|\boldsymbol{r}| \beta(\boldsymbol{r})\rangle \\
& +\sum_{\alpha} C_{v \alpha}^{*}(\boldsymbol{k}) C_{c \alpha}\left(\boldsymbol{k}^{\prime}\right) \delta_{\boldsymbol{k}, k^{\prime}} \boldsymbol{R} .
\end{aligned}
$$

The coefficients $(C)$ are components of each eigenvector, i.e., $\mathrm{N}_{\text {orbit }}$ complex numbers in general. The indices of $C$, i.e., $\alpha$ and $\beta$, span the 10 orbitals for $\mathrm{Si}$ and one orbital for $\mathrm{H}$. The second term in the right hand side of Eq. (14) is simply the element by element multiplication of valence and conduction eigenvectors which are weighted by coordinates of the atoms $\boldsymbol{R}$ in a unit cell. The first term on the right hand side of Eq. (14) contains the overlap integral between different types of orbitals on the same atom, i.e., $\langle\alpha(\boldsymbol{r})|\boldsymbol{r}| \beta(\boldsymbol{r})\rangle$ and it is called intra-atomic contribution. This quantity is an integral similar to Eq. (1) in which both radial and angular parts of each orbital must be used to find the overlap value. Since many of the overlap integrals vanish due to symmetry, only 15 out of the 100 overlap integrals are non-zero and need to be calculated. In this work, we assume Slater type orbitals ${ }^{77}$

$$
\phi(r)=N r^{n^{*}-1} e^{-\frac{Z-s}{n^{*}}} .
$$

$\mathrm{Z}$ is the atomic number, $s$ is a screening constant, and $n^{*}$ is effective quantum number. $\mathrm{N}$ is the normalization factor which is calculated by normalizing the whole orbital (both angular and radial parts included). We verified that our orbitals match with the orbitals reported in Ref. 75 for the more restricted $\mathrm{sp}^{3} \mathrm{~s}^{*}$ TB method. However, here we also include $3 \mathrm{~d}$ and $4 s^{*}$ orbitals following the procedure in Ref. 78 wherein the authors used $d^{*}$ for III-V semiconductors. Table I lists the values of $n^{*}, Z-s$, radial and angular parts of orbitals calculated for a silicon atom.

The values of fifteen non-zero overlap integrals for silicon are given in Table II. Integration over the radial part is found by numerical integration. For the angular part, the exact integration of spherical harmonics is found analytically. ${ }^{79}$ The angular part of the orbitals or $\mathrm{Y}_{1 \mathrm{~m}}$, which are written in Table I, can be found in the literature. ${ }^{80}$ In our

TABLE I. Slater type orbitals for a silicon atom.

\begin{tabular}{|c|c|}
\hline Non-zero overlap integrals & Value $(\AA)$ \\
\hline$\left\langle\mathrm{s}|\mathrm{z}| \mathrm{p}_{\mathrm{z}}\right\rangle=\left\langle\mathrm{s}|\mathrm{x}| \mathrm{p}_{\mathrm{x}}\right\rangle=\left\langle\mathrm{s}|\mathrm{y}| \mathrm{p}_{\mathrm{y}}\right\rangle$ & 1.4636 \\
\hline$\left\langle\mathrm{s}^{*}|\mathrm{z}| \mathrm{p}_{\mathrm{z}}\right\rangle=\left\langle\mathrm{s}^{*}|\mathrm{x}| \mathrm{p}_{\mathrm{x}}\right\rangle=\left\langle\mathrm{s}^{*}|\mathrm{y}| \mathrm{p}_{\mathrm{y}}\right\rangle$ & 0.3697 \\
\hline$\left\langle\mathrm{p}_{\mathrm{z}}|\mathrm{z}| \mathrm{d}_{3 \mathrm{z}_{-\mathrm{r}}^{2}}^{2}\right\rangle$ & 0.4029 \\
\hline$\left\langle\mathrm{p}_{\mathrm{x}}|\mathrm{z}| \mathrm{d}_{\mathrm{zx}}\right\rangle=\left\langle\mathrm{p}_{\mathrm{y}}|\mathrm{z}| \mathrm{d}_{\mathrm{yz}}\right\rangle$ & 0.3525 \\
\hline$\left\langle\mathrm{p}_{\mathrm{x}}|\mathrm{x}| \mathrm{d}_{\mathrm{x}-\mathrm{y}}{ }^{2}\right\rangle=\left\langle\mathrm{p}_{\mathrm{y}}|\mathrm{x}| \mathrm{d}_{\mathrm{xy}}\right\rangle=\left\langle\mathrm{p}_{\mathrm{z}}|\mathrm{x}| \mathrm{d}_{\mathrm{zx}}\right\rangle$ & 0.3525 \\
\hline$\left\langle\mathrm{p}_{\mathrm{y}}|\mathrm{y}| \mathrm{d}_{\mathrm{x}-\mathrm{y}}{ }^{2}\right\rangle=\left\langle\mathrm{p}_{\mathrm{x}}|\mathrm{y}| \mathrm{d}_{\mathrm{xy}}\right\rangle=\left\langle\mathrm{p}_{\mathrm{z}}|\mathrm{y}| \mathrm{d}_{\mathrm{yz}}\right\rangle$ & 0.3525 \\
\hline
\end{tabular}

\begin{tabular}{lcccc}
\hline \hline Orbital index & Orbital type & $\mathrm{n}^{*}$ & $\mathrm{Z}$-s & Radial and angular part \\
\hline 1 & $\mathrm{~s}$ & 3 & 4.15 & $0.37032 \mathrm{r}^{2} \mathrm{e}^{-1.383 \mathrm{r}}$ \\
2 & $\mathrm{p}_{\mathrm{x}}$ & 3 & 4.15 & $0.37032 \mathrm{r}^{2} \mathrm{e}^{-1.383 \mathrm{r}} \sin \theta \cos \phi$ \\
3 & $\mathrm{p}_{\mathrm{y}}$ & 3 & 4.15 & $0.37032 \mathrm{r}^{2} \mathrm{e}^{-1.383 \mathrm{r}} \sin \theta \sin \phi$ \\
4 & $\mathrm{p}_{\mathrm{z}}$ & 3 & 4.15 & $0.37032 \mathrm{r}^{2} \mathrm{e}^{-1.383 \mathrm{r}} \cos \theta$ \\
5 & $\mathrm{~d}_{\mathrm{xy}}$ & 3 & 1 & $0.0049 \mathrm{r}^{2} \mathrm{e}^{-0.3333 \mathrm{r}} \sin ^{2} \theta \sin 2 \phi$ \\
6 & $\mathrm{~d}_{\mathrm{yz}}$ & 3 & 1 & $0.0098 \mathrm{r}^{2} \mathrm{e}^{-0.3333 \mathrm{r}} \sin \theta \cos \theta \sin \phi$ \\
7 & $\mathrm{~d}_{\mathrm{zx}}$ & 3 & 1 & $0.0098 \mathrm{r}^{2} \mathrm{e}^{-0.3333 \mathrm{r}} \sin \theta \cos \theta \cos \phi$ \\
8 & $\mathrm{~d}_{\mathrm{x}}^{2}{ }^{2}{ }^{2}$ & 3 & 1 & $0.0049 \mathrm{r}^{2} \mathrm{e}^{-0.3333 r} \sin { }^{2} \theta \cos 2 \phi$ \\
9 & $\mathrm{~d}_{3 \mathrm{z}^{2}-\mathrm{r}}^{2}$ & 3 & 1 & $0.0028 \mathrm{r}^{2} \mathrm{e}^{-0.3333 \mathrm{r}}\left(3 \cos { }^{2} \theta-1\right)$ \\
10 & $\mathrm{~s}^{*}$ & 3.7 & 1.45 & $0.49 \times 10^{-4} \mathrm{r}^{2.7} \mathrm{e}^{-0.3919 \mathrm{r}}$ \\
\hline \hline
\end{tabular}

TABLE II. Non-zero overlap integrals for Slater type orbitals in silicon.

case, $l$ spans 0,1 , and 2 , and $\mathrm{m}$ spans 0 for $s$ and $s^{*}$ orbitals, $\{-1,0,+1\}$ for three $p$ orbitals and $\{-2,-1,0,+1,+2\}$ for five $d$ orbitals.

\section{Numerical method}

The one-dimensional band structure of SiNWs allows further simplification of Eq. (11) for numerical calculation. In case of a nanowire, the wave vector $\boldsymbol{k}$ is a $1 \mathrm{D}$ vector along the axis of the SiNWs (z-axis), i.e., $\boldsymbol{k}=k \hat{z}$. The summation over $\mathbf{k}$ is reduced to

$$
\sum_{k} \ldots=2 \times \frac{L_{n w}}{2 \pi} \int_{1 D B Z} \ldots d k,
$$

where $\mathrm{L}_{\mathrm{nw}}$ is the length of the nanowire and the extra factor of 2 takes the spin degeneracy into account. Let $\mathrm{E}_{\mathrm{cv}}(\boldsymbol{k})=\mathrm{E}_{\mathrm{c}}(\boldsymbol{k})$ - $\mathrm{E}_{\mathrm{v}}(\boldsymbol{k})$. Now Dirac's delta function can be expanded using the roots $\left(\mathrm{K}_{\mathrm{zp}}\right)$ of the equation $E_{c v}\left(\boldsymbol{k}_{z p}\right)-\hbar \omega=0$. That is,

$$
\delta\left(E_{c v}(k)-\hbar \omega\right)=\sum_{K_{z p}} \frac{\delta\left(k-K_{z p}\right)}{\left|\frac{\partial E_{c v}(\boldsymbol{k})}{\partial k}\right|_{@ K_{z p}}} .
$$

Using Eq. (17), the dielectric function [Eq. (11)] can be written as

$$
\epsilon_{2}(\omega)=\frac{e^{2}}{\epsilon_{0} m_{0}^{2} A_{n w} \omega^{2}} \sum_{c, v} \int_{k}\left|\left\langle u_{c}|\hat{e} . \boldsymbol{p}| u_{v}\right\rangle\right|^{2} \sum_{K_{z p}} \frac{\delta\left(k-K_{z p}\right)}{\left|\frac{\partial E_{c v}(k)}{\partial k}\right|_{@ K_{z p}}} d k .
$$

By interchanging the integration and the last summation and using the sifting property of Dirac's delta, Eq. (18) becomes

$$
\epsilon_{2}(\omega)=\frac{2 e^{2}}{\epsilon_{0} m_{0}^{2} A_{n w} \omega^{2}} \sum_{c, v} \sum_{K_{z p} \geq 0} \frac{\left|\left\langle u_{c}|\hat{e} \cdot \boldsymbol{p}| u_{v}\right\rangle\right|^{2}}{\left|\frac{\partial E_{c v}(k)}{\partial k}\right|_{@ K_{z p} \geq 0}},
$$

where $A_{n w}$ is the cross-sectional area of the nanowire. From the calculation of the band structure and Eigen states, all possible combinations of valence to conduction transitions (i.e., $\mathrm{E}_{\mathrm{cv}}$ and corresponding matrix elements $\left.\left\langle u_{c}|\hat{e} \cdot \boldsymbol{p}| u_{v}\right\rangle\right)$ are found for each given $k$ value along the $\mathrm{BZ}\left(\mathrm{k}_{\mathrm{z}}\right.$-axis) [See Fig. 2(b)]. The method of implementing Eq. (19) is depicted in Fig. 2(b) for an example in which the band structure has 2 and 3 valence and conduction bands, respectively. If the band structure has 100 points along the $\mathrm{BZ}$, then it is required to store 600 different values for $\mathrm{E}_{\mathrm{cv}}$ and optical matrix elements. Now at each given value of frequency $(\omega)$, the number of $\boldsymbol{K}_{z \boldsymbol{p}}$ 
points at which $\mathrm{E}_{\mathrm{cv}}\left(\boldsymbol{K}_{z p}\right)=\hbar \omega$ is found by counting the number of times the modified band structure, $\mathrm{E}_{\mathrm{cv}}(\boldsymbol{k})$, crosses the horizontal $\hbar \omega$ line [Fig. 2(b)].

The absorption, $\alpha(\omega)$, is calculated using the extinction ratio, $\kappa(\omega)$. The extinction ratio can be expressed in terms of real and imaginary parts of dielectric function, i.e., $\varepsilon_{1}(\omega)$ and $\varepsilon_{2}(\omega)$, respectively. Since the imaginary part of the dielectric function has already been calculated, it is possible to find the real part by applying Kramers-Krönig theorem. ${ }^{81}$ We first show how this helps in the numerical evaluation of $\varepsilon_{1}(\omega)$. To show this, Eq. (11) is directly inserted into the KramersKrönig integral as follows:

$$
\begin{aligned}
\epsilon_{1}\left(\omega^{\prime}\right)= & 1+\frac{2}{\pi} P\left[\int_{0}^{\infty} \frac{\varepsilon_{2}(\omega) \omega d \omega}{\omega^{2}-\omega^{\prime 2}}\right]=1+\frac{2}{\pi} \\
& P\left[\int_{0}^{\infty} \frac{\pi e^{2}}{\epsilon_{0} m_{0}^{2} V \omega^{2}} \sum_{c, v} \sum_{k}\left|\left\langle u_{c}|\hat{e} \cdot \boldsymbol{p}| u_{v}\right\rangle\right|^{2}\right. \\
& \left.\times \delta\left(E_{c v}(\boldsymbol{k})-\hbar \omega\right) \frac{\omega d \omega}{\omega^{2}-\omega^{\prime 2}}\right]=1+\frac{2 e^{2}}{\epsilon_{0} m_{0}^{2} V} \\
& \times \sum_{c, v} \sum_{k}\left|\left\langle u_{c} \mid \hat{e} \cdot \overline{\boldsymbol{P} \mid} u_{v}\right\rangle\right|^{2} \int_{0}^{\infty} \delta\left(E_{c v}(\boldsymbol{k})-\hbar \omega\right) \\
& \times \frac{\hbar^{2} d \hbar \omega}{\hbar \omega\left((\hbar \omega)^{2}-\left(\hbar \omega^{\prime}\right)^{2}\right)} .
\end{aligned}
$$

Using the sifting property of Dirac's delta function and converting the summation over $\mathrm{k}$ vectors to an integration lead to

$$
\begin{aligned}
\epsilon_{1}\left(\omega^{\prime}\right)= & 1+\frac{4 e^{2} \hbar^{2}}{\pi \epsilon_{0} m_{0}^{2} A_{n w}} \sum_{c, v} \int_{k=0}^{k=\pi} \\
& \times\left|\left\langle u_{c}|\hat{e} \cdot \boldsymbol{p}| u_{v}\right\rangle\right|^{2} \frac{d k}{E_{c v}(\boldsymbol{k})\left(\left(E_{c v}(\boldsymbol{k})\right)^{2}-\left(\hbar \omega^{\prime}\right)^{2}\right)} .
\end{aligned}
$$

Evaluating Eq. (21) requires three nested summation loops. The first loop runs over the incoming light frequencies $\left(\omega^{\prime}\right)$. The second loop runs over all combinations of valence to conduction band transitions $(c v)$ and the third loop runs over all discrete $k$ points along the BZ (i.e., $\mathrm{k}_{\mathrm{z}}=0$ to $\pi$ ). After this step, both real and imaginary parts of the dielectric function are used to find the extinction ratio $(\kappa)$ and refractive index (n) of SiNW according to

$$
\begin{aligned}
& \kappa=\sqrt{\left(\sqrt{\varepsilon_{1}^{2}+\varepsilon_{2}^{2}}-\varepsilon_{1}\right) / 2} \text { and } \\
& n=\sqrt{\left(\sqrt{\varepsilon_{1}^{2}+\varepsilon_{2}^{2}}+\varepsilon_{1}\right) / 2 .}
\end{aligned}
$$

The relation between the absorption coefficient and the extinction ratio is given as follows: ${ }^{81}$

$$
\alpha(\omega)=\frac{4 \pi \kappa(\omega)}{\lambda_{0}}=\frac{4 \pi \kappa(\omega)}{c / f}=\frac{2 \omega \kappa(\omega)}{c} .
$$

Implementing Eqs. (19) and (21) requires storing all combinations of valence to conduction transitions (i.e., storing the corresponding energy differences and matrix elements) which renders the fully numerical method very slow, especially for large diameter nanowires. A simpler semi-analytical formulation based on effective mass approximation (EMA) is presented in the Appendix which offers a good approximation of absorption in a computationally more efficient manner. Note that in the numerical implementation of the equations in both Secs. II B 1 and II B 2 we use the right hand side of Eq. (13) to find the momentum matrix elements.

\section{Absorption in bulk silicon}

As a validation step, we will show that the tight binding method can successfully reproduce the experimental as well as DFT-based absorption spectrum of bulk silicon for all possible direct valence-to-conduction inter-band transitions.

To speed up the integration over the 3D BZ of bulk silicon, the calculation of Eigen states and absorption can be confined to an irreducible wedge as shown in Fig. 3. The wedge is $1 / 48$ th of the whole BZ; hence, the calculated quantities within this wedge must be weighted correctly. The higher the number of $\left(\mathrm{k}_{\mathrm{x}}, \mathrm{k}_{\mathrm{y}}, \mathrm{k}_{\mathrm{z}}\right)$ points (samples) taken from the wedge, the more precise the calculated absorption value is.

To calculate the Eigen states and Eigen energies, a discretized 3D k-space is built by dividing the 3D space of $(0,2 \pi / a) \times(0,2 \pi / a) \times(0,2 \pi / a)$ into $N_{x} \times N_{y} \times N_{z}$ points where $a$ is the lattice constant of silicon. Then, a search routine is used to find those $\left(\mathrm{k}_{\mathrm{x}}, \mathrm{k}_{\mathrm{y}}, \mathrm{k}_{\mathrm{z}}\right)$ points from this space which are surrounded by the five faces of the wedge which are given by the following equations: ${ }^{82}$

$k_{x}+k_{y}+k_{z}=\frac{3}{2} \frac{2 \pi}{a}, \quad k_{x}=k_{z}, k_{y}=k_{z}, k_{z}=\frac{2 \pi}{a}$ and $k_{z}=0$.

Now $\mathrm{N}_{\mathrm{w}}$ triplets of $\left(\mathrm{k}_{\mathrm{x}}, \mathrm{k}_{\mathrm{y}}, \mathrm{k}_{\mathrm{z}}\right)$ are found within the wedge which are saved for the next step of Eigen state/energy calculation. Using Bloch's theorem, we obtain the $20 \times 20$ matrix (two atoms per primitive cell), whose eigen values and functions correspond to the energy levels and wave functions. ${ }^{83}$ The lowest 4 Eigen-states and energies belong to the valence band and the rest (16 states) compose the conduction band.

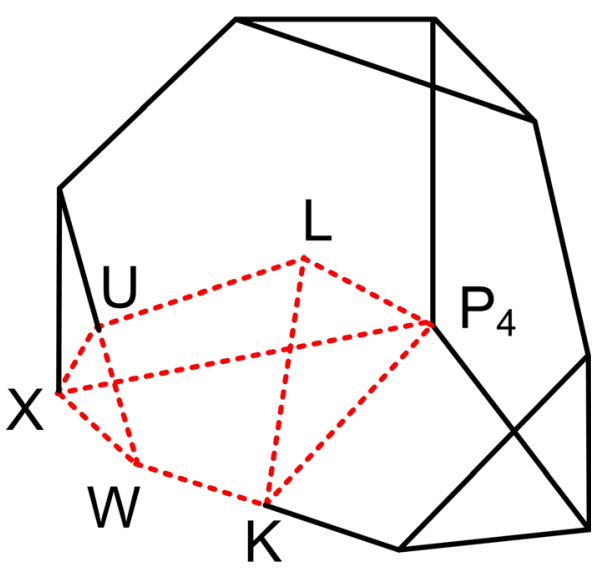

FIG. 3. The first BZ of the reciprocal lattice for bulk silicon with emphasis on the first octant (solid line) which contains the first irreducible wedge (dashed line). 
Hence, there are 64 possible valence to conduction band transitions at each given $k$ point within the 3D irreducible wedge [dashed lines in Fig. 3].

Afterwards, Eq. (11) is used to find the imaginary part of the dielectric function. Replacing the summation over $\boldsymbol{k}$ with 3D integration and using the Lorentzian broadening function to approximate the Dirac delta function yields

$$
\begin{aligned}
\epsilon_{2}(\omega)= & \frac{\pi e^{2}}{\epsilon_{0} m_{0}^{2} V \omega^{2}} \sum_{c, v} \frac{2 \times V \times 48}{8 \pi^{3}} \\
& \times \int_{\overrightarrow{\boldsymbol{K}}}\left|\left\langle u_{c}|\hat{\boldsymbol{e}} \cdot \boldsymbol{p}| u_{v}\right\rangle\right|^{2} \frac{\gamma / \pi}{\left(E_{c v}(\boldsymbol{k})-\hbar \omega\right)^{2}+\gamma^{2}} d^{3} k,
\end{aligned}
$$

where $\gamma$ is the broadening with a value of $10-20 \mathrm{meV}$ cho$\operatorname{sen}^{70}$ and the volume element is $d^{3} k=d k_{x} d k_{y} d k_{z}$.

Implementing Eq. (25) requires three nested loops corresponding to the photon energy $(\omega), 64$ combinations of sub bands $(c v)$, and $\mathrm{N}_{\mathrm{w}}$ values of $\boldsymbol{k}$ triplets within the wedge. The Kramers-Krönig code ${ }^{84}$ is used to find the real part of the dielectric function from Eq. (25). The total absorption is found by averaging three values corresponding to three different polarizations $(\mathrm{x}, \mathrm{y}, \mathrm{z})$. In parallel with the abovementioned procedure, the DFT method implemented in SIESTA is used to calculate the dielectric function, $\varepsilon_{2}(\omega)$, of bulk silicon. The results were post processed using available Fortran codes in SIESTA to find $\varepsilon_{1}(\omega)$ as well as $\alpha(\omega) .{ }^{57}$

\section{RESULTS}

\section{A. Direct transitions in bulk silicon}

The absorption spectrum of bulk silicon is calculated by $\mathrm{TB}$ and it is compared with experimental data ${ }^{85}$ in Fig. 4. Firstly, it is observed that the TB-based absorption spectrum starts at $3.24 \mathrm{eV}$ which is consistent with the value of the direct bandgap in bulk silicon. However, the DFT-based spectrum starts at a lower value due to underestimation of the bandgap with the LDA functional. Secondly, the close match

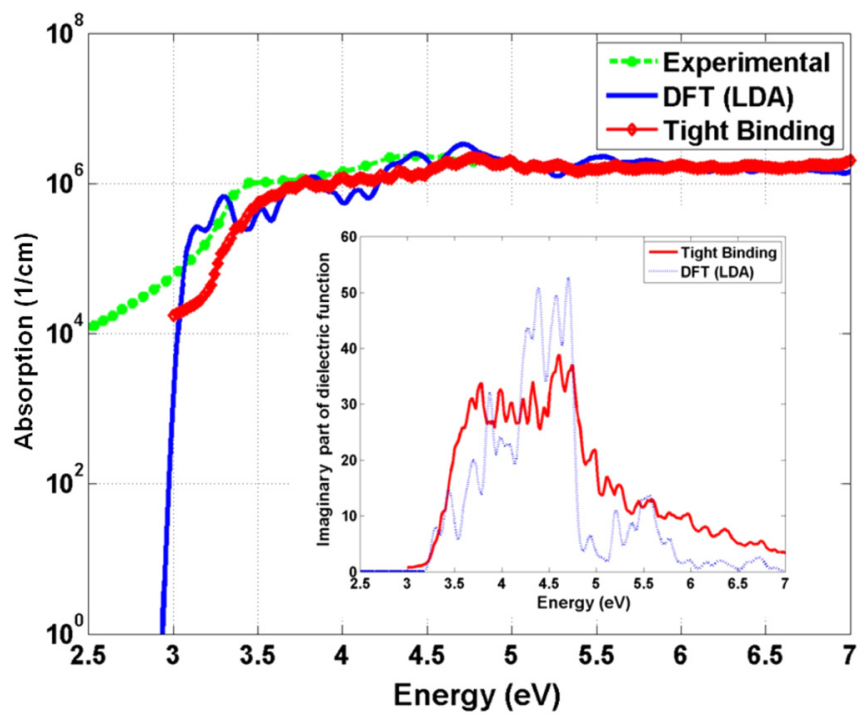

FIG. 4. Absorption spectrum of bulk silicon was calculated by DFT and TB. A comparison was made with experimental data. ${ }^{85}$ Inset: Imaginary part of dielectric function of bulk silicon, $\varepsilon_{2}(\omega)$, calculated by TB and DFT methods. between the experimental data, TB, and DFT results (e.g., $\alpha \approx 10^{6} \mathrm{~cm}^{-1}$ for $\mathrm{E}_{\mathrm{g}}>3.5 \mathrm{eV}$ ) lends some validation for application of the tight binding method for studying the light absorption in SiNWs. The low energy tail $(\mathrm{E}<3.24 \mathrm{eV})$ of the absorption spectrum which was calculated by the TB method is a numerical artifact and it has no physical meaning. This arises because the low energy skirts of many Lorentzian functions [last term of Eq. (25)] were being added together, and this resulted in a non-zero value. The inset of Fig. 4 shows the imaginary part of dielectric function, $\varepsilon_{2}(\omega)$, calculated by TB and DFT methods. Additionally, the static dielectric constant for bulk silicon using the TB method is found to be 10.1055 in close agreement with the experimental value of 11.9 at room temperature ${ }^{86}$ and values calculated using the TB method with different numbers of orbitals. ${ }^{68}$

\section{B. Absorption spectrum of [110] SiNWs}

Figure 5 compares the absorption spectra calculated using the fully numerical method (Sec. II B) and the semianalytical approach based on effective mass theory (see Appendix). Using the semi-analytical method significantly enhances the computational speed at the price of losing some precision at higher energies. The calculation time is reduced to less than one tenth of the time taken by the fully numerical method. Also, it is evident that the smaller diameter nanowire $(\mathrm{d}=0.5 \mathrm{~nm})$ has a larger bandgap $\left(\mathrm{E}_{\mathrm{g}}=3.6 \mathrm{eV}\right)$ than the larger diameter one $(\mathrm{d}=1.1 \mathrm{~nm})$ which has a bandgap of $2.25 \mathrm{eV}$. This is expected due to quantum confinement.

In terms of accuracy, it is apparent from Fig. 5 that the semi-analytical approach can produce comparable results, especially for energy values below $4.5 \mathrm{eV}$. This is because the $\mathrm{E}_{\mathrm{cv}}(k)$ sub bands fit very well to EMA-based parabola for this energy range. For higher photon energies, the sub bands tend to have a different or even negative curvature; however, some of the peak positions continue to be similar.

\section{Effect of anisotropy and diameter}

As it was observed theoretically ${ }^{45,87}$ and experimentally, ${ }^{88,89}$ nanowires have strong optical anisotropy. For

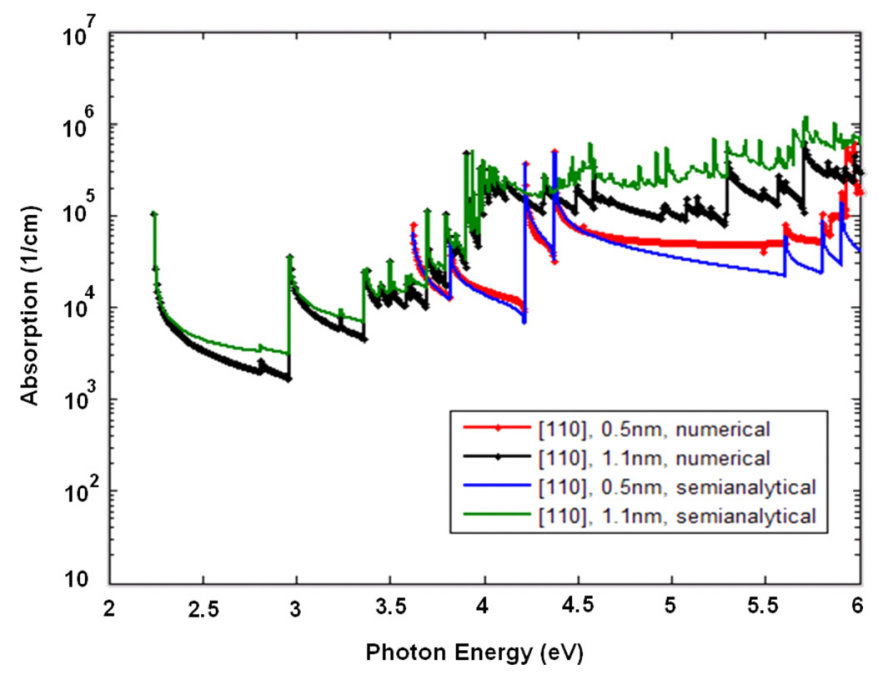

FIG. 5. Comparison of the absorption spectra for $0.5 \mathrm{~nm}$ and $1.1 \mathrm{~nm}$ [110] SiNWs, calculated by fully numerical and semi-analytical methods. 


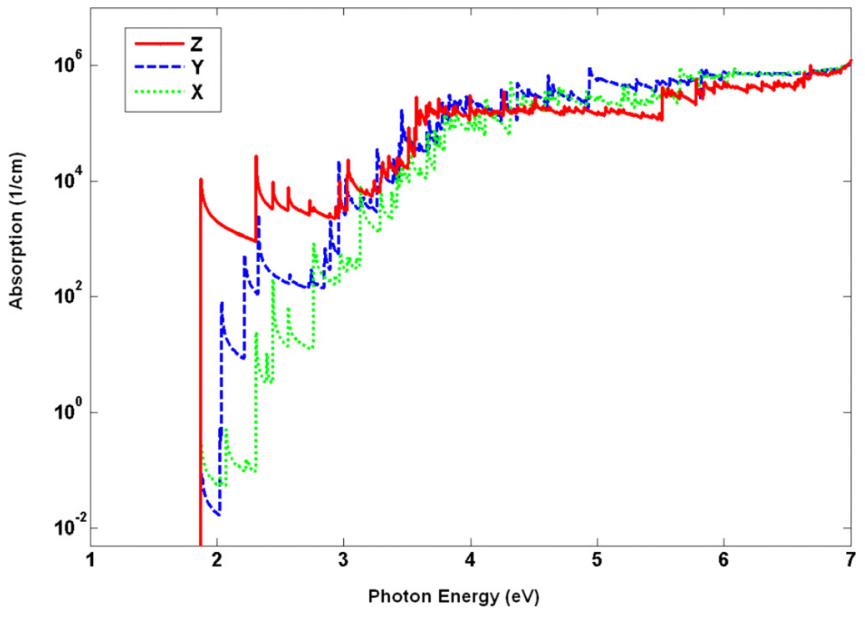

FIG. 6. Absorption spectrum of a $1.7 \mathrm{~nm}$ [110] SiNW for the three different incident photon polarizations.

example, in [110] silicon nanowires, the photoluminescence is stronger when polarization is parallel to the nanowire axis. The same effect is observed here in the band edge absorption spectrum of [110] SiNWs. As Fig. 6 shows, in a $1.7 \mathrm{~nm}$ [110] SiNW, the absorption value for z-polarized photons is 6 orders of magnitude higher than the same quantity for $\mathrm{x}$ and y polarizations. In photo detectors made from SiNWs, this effect could make the photocurrent highly sensitive to polarization of incident light.

In our previous work, ${ }^{45}$ we observed that the optical dipole matrix elements for z-polarized photons had a stronger role in determining the value of spontaneous emission time in $2.3 \mathrm{~nm}$ and $3.1 \mathrm{~nm}$ [110] SiNWs. This means that the value of absorption for $\mathrm{z}$-polarized photons is also larger for these SiNWs. We also notice that absorption is inversely proportional to the diameter of the nanowire, due to the decrease of the optical dipole matrix element with the increase in diameter (Fig. 7). The change of the matrix element with diameter (confinement) has been previously explained using the particle in a box model. ${ }^{70}$

For the sake of clarity, the absorption spectra in Fig. 7 is magnified around the band edge in order to show the exact difference between $1.7 \mathrm{~nm}, 2.3 \mathrm{~nm}$, and $3.1 \mathrm{~nm}$ [110] SiNWs.

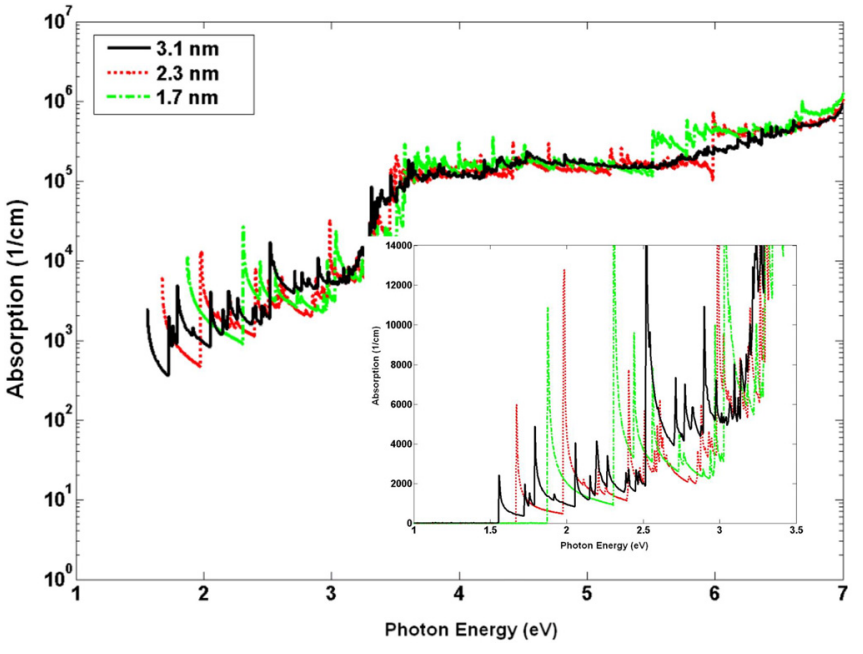

FIG. 7. Absorption spectrum of three SiNWs with diameters of $1.7 \mathrm{~nm}$, $2.3 \mathrm{~nm}$ and $3.1 \mathrm{~nm}$. The photon polarization is along the z-axis. The inset shows the magnified section of the absorption spectra around the bandgap energy.

We observe in the inset that the first absorption peak belongs to a $3.1 \mathrm{~nm}$ [110] SiNW. It has the smallest band edge absorption compared to the other nanowires considered. This result suggests that embedding arrays of silicon nanowires covering a wide range of diameters can broaden the absorption spectrum of the host material as each array is sensitive to different cut-off wavelengths as a result of its bandgap.

\section{Effect of strain}

Effect of strain on the spontaneous emission of photons in nanowires was explained through the change of the optical transition matrix element arising from the change of wave function symmetry. ${ }^{45}$ The symmetry of wave functions was almost intact in the tensile strain regime which resulted in very close values of spontaneous emission time for this regime. On the other hand by entering into compressive strain regime, the spontaneous emission time decreased by a few orders of magnitude. This is due to the valence band flip which changed the wave function symmetry.

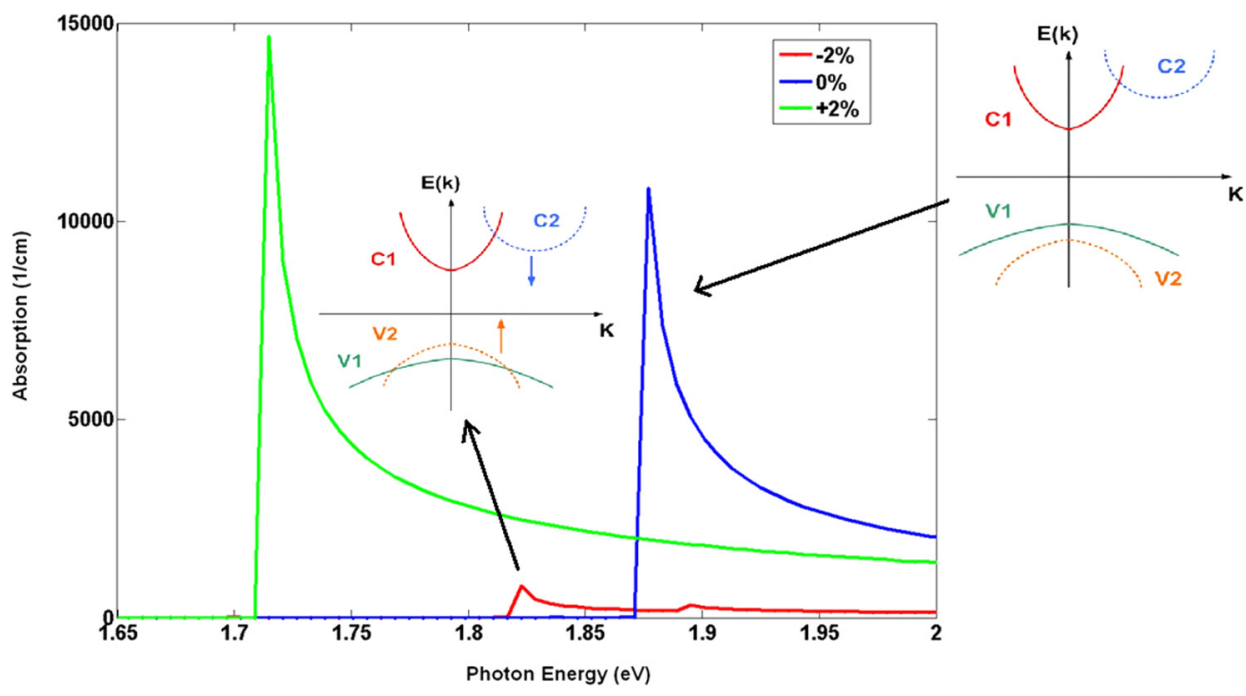

FIG. 8. Absorption spectra for $-2 \%$, $0 \%$, and $+2 \%$ strained $1.7 \mathrm{~nm}$ [110] SiNWs. Incident photons are $\mathrm{z}$ polarized in all cases. At $-2 \%$ strain, the interchange of $\mathrm{V} 1$ and $\mathrm{V} 2$ reduces the matrix element, as V2 has different symmetry than V1. 


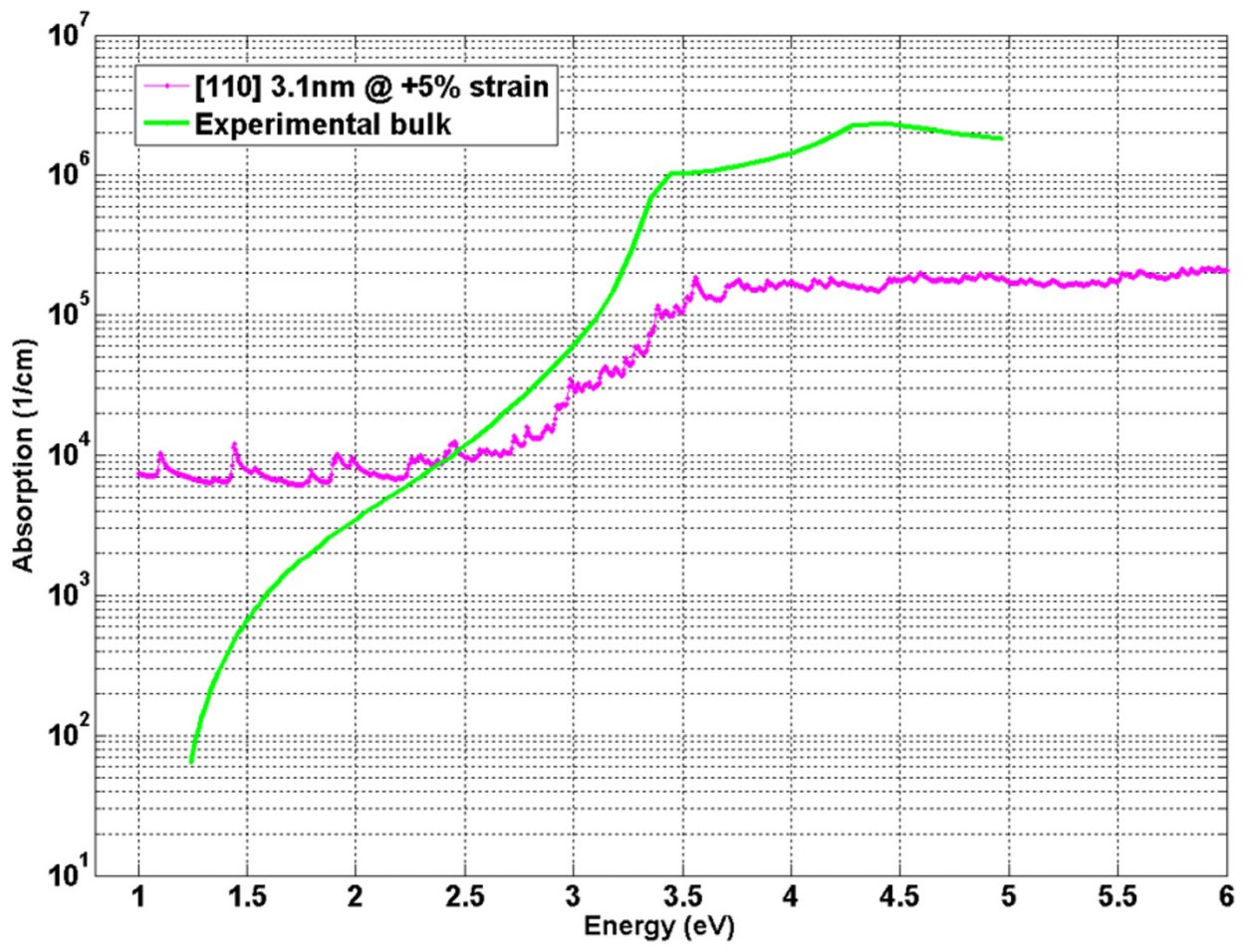

FIG. 9. Comparison of the bulk silicon absorption spectrum (experimental ${ }^{85}$ with an indirect bandgap of $1.1 \mathrm{eV}$ ) with that of a $3.1 \mathrm{~nm} \mathrm{[110]} \mathrm{SiNW}$ at $+5 \%$ strain (tight binding calculation with a direct bandgap of 1.1. eV).

In the context of absorption, we show that this mechanism causes a large variation of band edge absorption in the compressive strain regime. Figure 8 shows the absorption spectra of a $1.7 \mathrm{~nm}[110] \mathrm{SiNW}$ at $-2 \%, 0 \%$, and $+2 \%$ strain values. As it is evident, the values of absorption for $0 \%$ and $+2 \%$ strained nanowires are fairly close to each other; however for the compressively strained nanowire $(-2 \%)$, we observe one order of magnitude drop in the value of absorption. This is due to the valence band interchange that occurs in response to compressive strain. As shown in Fig. 8, the valence sub band V2 which had lower energy than V1 in case of $0 \%$ and $+2 \%$ strain is now shifted upward as a result of its anti-bonding orbital composition in response to $-2 \%$ strain. ${ }^{34,48,51}$ Hence due to its different symmetry compared to V1, it lowers the value of the optical matrix element compared with other cases in which $\mathrm{C} 1$ and V1 determine the band edge transition rate. The normalized momentum matrix element values for $0 \%$ and $+2 \%$ strained nanowires are $\sim 15 \mathrm{meV}$ at the $\mathrm{BZ}$ center, while for $-2 \%$ strained nanowire it is $1.5 \mathrm{meV}$. This can explain the one order of magnitude difference between absorption values at $0 \%$ and $-2 \%$ strains.

Figure 9 compares the experimental absorption spectrum of bulk silicon ${ }^{85}$ and the calculated spectrum for a $3.1 \mathrm{~nm}$ [110] SiNW under $+5 \%$ strain. Since the unstrained

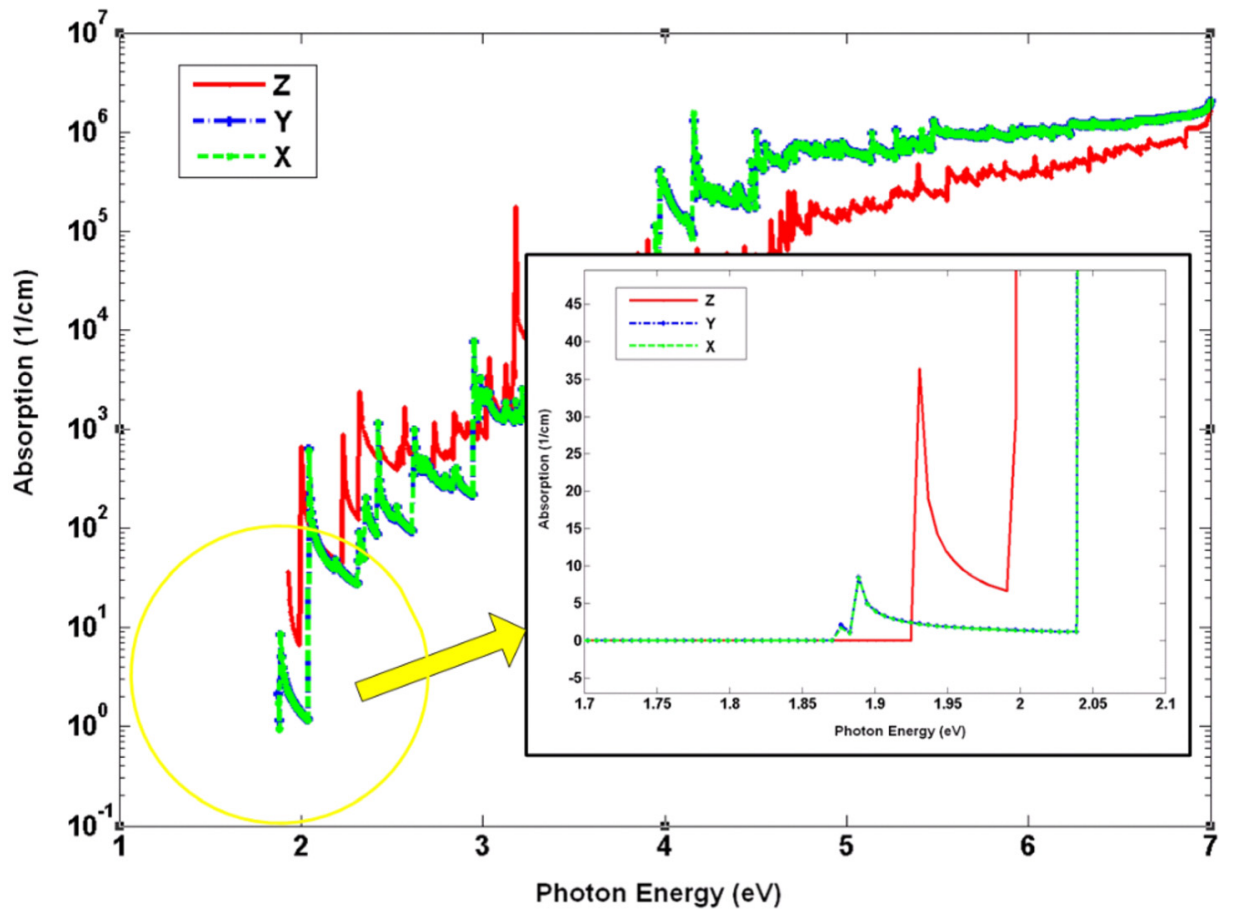

FIG. 10. Absorption spectrum of a $2.2 \mathrm{~nm}$ [100] SiNW. The inset shows that the band edge absorption is nonzero for $\mathrm{x}$ and $\mathrm{y}$ polarized photons, while it is zero for the $\mathrm{z}$ polarized case. 
bandgap of [110] nanowires in this work is more than that of bulk silicon, a strained nanowire is chosen for comparison purpose. The nanowire under study has a direct bandgap of $1.1 \mathrm{eV}$ as opposed to bulk silicon which has an indirect bandgap at 1.1.eV; hence, a higher absorption is expected for the nanowire provided the dipole matrix elements are nonzero. It is assumed that the incident light is polarized along the $\mathrm{z}$-axis. We see in Fig. 9 that in the energy range of $1-2 \mathrm{eV}$, the nanowire has a greater amount of absorption than bulk silicon, i.e., $7000 \mathrm{~cm}^{-1}$ versus $100 \mathrm{~cm}^{-1}$. Within this range, the absorption process in bulk silicon is a weak second order process mediated by phonons.

\section{Absorption spectrum of [100] SiNWs}

Comparing the absorption spectrum of [110] and [100] SiNWs is also instructive since it further reveals the role of
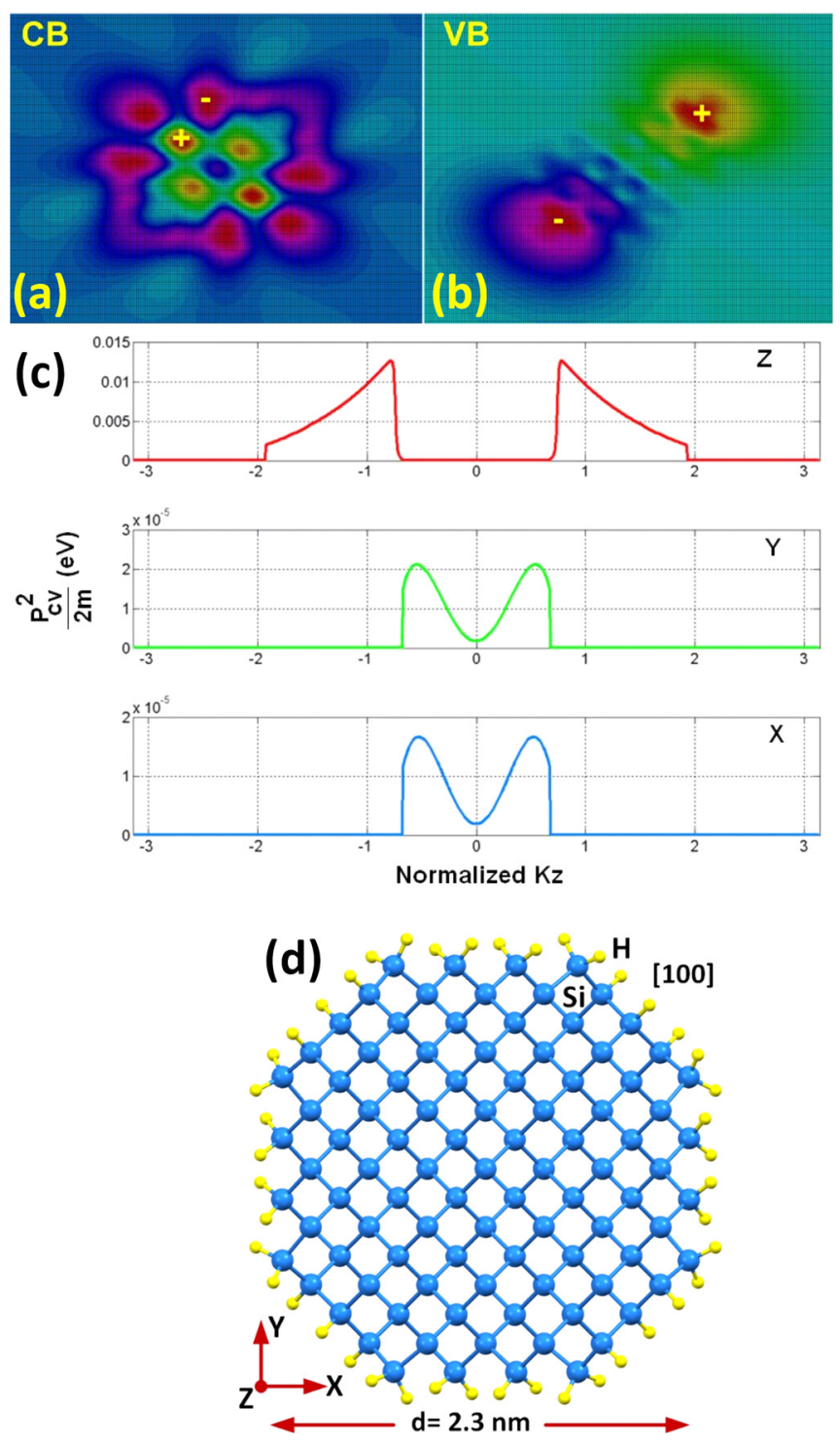

FIG. 11. Symmetry of the conduction (a) and valence (b) sub bands at the BZ center reveals centro-symmetric and noncentro-symmetric nature. (c) The normalized value of the momentum matrix element in terms of eV along 1D BZ $\left(-\pi<\mathrm{k}_{\mathrm{z}}<\pi\right)$ for $\mathrm{x}, \mathrm{y}$, and $\mathrm{z}$ polarized photons. (d) The cross sectional view of $2.2 \mathrm{~nm}$ [100] SiNW. The band structure of this nanowire is shown in Fig. 1(b). wave function symmetry in determining the optical anisotropy. Figure 10 shows the absorption spectrum of a $2.2 \mathrm{~nm}$ [100] unstrained SiNW in a wide energy range including the magnified portion near the band edge (Inset). As it can be seen here, the band edge absorption is predominantly determined by $\mathrm{x}$ and $\mathrm{y}$ polarizations. For the $\mathrm{z}$ polarized case, the matrix element (and hence absorption) vanishes (Fig. 10).

The wave functions corresponding to conduction/valence band minimum/maximum (at BZ center) are plotted in the xy cross sectional plane of the nanowire in Figs. 11(a) and 11(b), respectively. The conduction and valence bands have even and odd symmetry with respect to the center of rotation, respectively. Recalling the definition of the position operator matrix element, i.e., Eq. (1), we observe that the integrand must have an even parity in order to obtain a nonzero matrix element. Since $\mathrm{x}$ and y operators have odd parity, they both make the integrand to be of even symmetry. As a result, the $x$ and y polarized matrix elements are nonzero as opposed to the z-polarized matrix element. Since the cross section of the nanowire has $\mathrm{C} 4$ group symmetry, the $\mathrm{z}$ operator is of A type which results in an integrand of odd symmetry, and hence, a zero value for the matrix element is expected. Figure 11(c) shows the normalized momentum matrix element along the $\mathrm{BZ}$ of the nanowire in which $\mathrm{x}$ - and $\mathrm{y}$ - polarization components are more dominant than the z-component. Figure 11(d) shows the symmetry of the nanowire cross section, which suggests that the $\mathrm{x}$ - and $\mathrm{y}$ - components of dipole matrix elements are comparable. A similar anisotropy was observed in DFT + LDA calculations performed on a [100] SiNW with a diameter of $\sim 1 \mathrm{~nm} .{ }^{87}$

\section{CONCLUSIONS}

The absorption spectra of SiNWs were calculated using a semi-empirical ten orbital tight binding method. Results for the absorption spectra from this method were compared to results from DFT calculations and experimental data, for direct transitions in bulk silicon. After detailed explanations of both TB-based fully numerical and EMA-based semi-analytical methods, and demonstrating their agreement in calculating the absorption spectrum, we investigated the effect of diameter, crystallographic direction, optical anisotropy, and mechanical strain on the absorption of SiNWs. It was observed that strain can change the band edge absorption by at least one order of magnitude due to the change in the symmetry of wave functions and optical matrix element. The optical anisotropy also manifests itself in the different values of band edge absorption corresponding to different polarizations of incident photons. For the case of hexagonal cross section [110] silicon nanowires, we observed high absorption for z-polarized photons. In contrast to this, square cross section [100] nanowires showed equally strong absorption for $\mathrm{x}$ and $\mathrm{y}$ polarized photons and zero (symmetry forbidden) absorption for $\mathrm{z}$ polarized photons. Vital for the application of SiNWs in solar cells we found that SiNWs can have 100 times larger absorption compared to bulk silicon in the same range of IR energy $\left(E_{\mathrm{g}}=1.1 \mathrm{eV}\right)$. We also observed that different diameters of silicon nanowires show different band edge absorption. This study suggests potential 
applications of silicon nanowires in spectrum widening of solar cells or photo detectors. The equivalence of the stimulated emission rate with absorption spectra facilitates the calculation of the gain spectrum by assuming a partially filled conduction band and partially empty valence band (population inversion). In this case, the difference of Fermi factors [last parenthesis of Eq. (9)] must be properly updated. Based on this, tuning of emission wavelength in nanowire-based lasers using diameter, strain, and crystallographic direction can be proposed.

\section{ACKNOWLEDGMENTS}

Daryoush Shiri acknowledges access to the supercomputing facilities provided by Shared Hierarchical Academic Research Computing Network (SHARCNET ${ }^{\circledR}$ ) in Ontario, Canada while working at University of Waterloo. The work of M. Golam Rabbani and M. P. Anantram was supported by the National Science Foundation under Grant numbers 1001174 and 1231927. M. P. Anantram was also partially supported by QNRF Grant (No. NPRP 5-9682-403) and the University of Washington.

\section{APPENDIX: SEMI-ANALYTICAL CALCULATION OF ABSORPTION SPECTRUM}

Equation (21) can be simplified by making the following assumptions: Firstly it is assumed that the band structure is of parabolic shape. Therefore, the energy of each band can be written in effective mass formalism as

$$
\begin{aligned}
& E_{c}(k)=E_{c \text { min }}+\frac{\hbar^{2} k^{2}}{2 m_{c}^{*}}, \\
& E_{v}(k)=E_{v \max }-\frac{\hbar^{2} k^{2}}{2 m_{v}^{*}} \rightarrow E_{c v}(k)=E_{g}+\frac{\hbar^{2} k^{2}}{2 \mu_{c v}},
\end{aligned}
$$

where $\mu_{c v}$ is the reduced effective mass of a conduction and valence band pair, i.e., $\mu_{c v}=\frac{m_{c}^{*} m_{v}^{*}}{m_{c}^{*}+m_{v}^{*}}$.

Secondly it is assumed that the optical dipole matrix element value around the BZ center is almost constant. As a result, for a given combination of bands (e.g., $c_{i}$ and $v_{j}$ ), the optical dipole matrix element for inter band transitions at each $k$ value is equal to the matrix element value given at $k=0$

$$
\left|\left\langle u_{c}|\hat{e} \cdot \boldsymbol{p}| u_{v}\right\rangle\right|^{2}=\left|\left\langle u_{c}|\hat{e} \cdot \boldsymbol{p}| u_{v}\right\rangle\right|_{@ k=0}^{2}=\left|\boldsymbol{p}_{c v}(k=0)\right|^{2} \text {. }
$$

With these assumptions, Eq. (19) of the main text is reduced to

$$
\epsilon_{2}(\omega)=\frac{2 e^{2}}{\epsilon_{0} m_{0}^{2} A_{n w} \omega^{2}} \sum_{c, v} \frac{\left|\boldsymbol{p}_{c v}(k=0)\right|^{2}}{\left|\frac{\hbar^{2} k}{2 \mu_{c v}}\right|}
$$

The summation over $k_{z p}$ is not required here since the horizontal line of $\hbar \omega$ always intersects each $\mathrm{E}_{c v}(k)$ at one point [See Fig. 2(b)]. The crossing due to the next $\mathrm{E}_{\mathrm{cv}}(\mathrm{k})$ is already taken into account in the summation over $c v$ in Eq. (A3). This equation can be simplified by replacing $k$ with the following value

$$
k=\frac{\sqrt{2 \mu_{c v}}}{\hbar} \sqrt{E_{c v}(k)-E_{g, c v}}=\frac{\sqrt{2 \mu_{c v}}}{\hbar} \sqrt{\hbar \omega-E_{g, c v}},
$$

where $E_{g, c v}$ represents the minimum of $\mathrm{E}_{c v}(k)$ [i.e., $\left.\mathrm{E}_{c v}(k=0)\right]$ for the corresponding $c v$ index. For example in Fig. 2(b), $E_{g, c v}$ spans six values; hence Eq. (A4) is further reduced to the following equation in which the summation adds the six terms sequentially

$$
\begin{aligned}
\epsilon_{2}(\omega) & =\frac{2 e^{2}}{\epsilon_{0} m_{0}^{2} A_{n w} \omega^{2}} \sum_{c, v} \frac{\left|\boldsymbol{p}_{c v}(k=0)\right|^{2} \sqrt{2 \mu_{c v}}}{\hbar \sqrt{\hbar \omega-E_{g, c v}}} \\
& =\frac{2 \sqrt{2} e^{2}}{\epsilon_{0} m_{0}^{2} A_{n w} \omega^{2} \hbar} \sum_{c, v} \frac{\left|\boldsymbol{p}_{c v}(k=0)\right|^{2} \sqrt{\mu_{c v}}}{\sqrt{\hbar \omega-E_{g, c v}}} .
\end{aligned}
$$

For each photon energy $\hbar \omega$, the summation runs only over all combinations of conduction and valence band $(c v)$ with $\mathrm{E}_{\mathrm{c}}-\mathrm{E}_{\mathrm{v}}=\hbar \omega$ and optical matrix elements at $k=0$. Therefore, this method is advantageous over the fully numerical method [Eq. (19)] if it can show that both methods give similar results. To obviate the singularities at $\hbar \omega=E_{g, c v}$, we can use the following approximation by introducing a broadening factor $^{70}$ of $\eta$

$$
\frac{1}{\sqrt{x}} \cong \frac{1}{\sqrt{x+i \eta}}=\frac{\sqrt{x-i \eta}}{\sqrt{x^{2}+\eta^{2}}} \cong \operatorname{real}\left(\frac{\sqrt{x-i \eta}}{\sqrt{x^{2}+\eta^{2}}}\right) \text {. }
$$

The real part of the dielectric function is found by inserting Eq. (A5) into the Kramers-Krönig integral which yields

$$
\begin{gathered}
\epsilon_{1}(\omega)=1+\frac{4 \sqrt{2} e^{2}}{\pi \epsilon_{0} m_{0}^{2} A_{n w} \omega^{2} \hbar} \sum_{c v}\left|\boldsymbol{P}_{c v}(k=0)\right|^{2} \sqrt{\mu_{c v}} \\
P\left[\int_{0}^{\infty} \frac{d \omega^{\prime}}{\omega^{\prime}\left(\omega^{\prime 2}-\omega^{2}\right) \sqrt{\omega^{\prime}-\left(E_{g, c v}\right) / \hbar}}\right] .
\end{gathered}
$$

The right hand side of Eq. (A7) can be further simplified using Wolfram Mathematica ${ }^{\circledR}$ online integrator ${ }^{79}$ or the code available in Ref. 84, and using the Kramers-Krönig integral. Calculating extinction ratio and absorption are then completed using Equations (22) and (23).

${ }^{1} \mathrm{~J}$. Xiang et al., "Ge/Si nanowire heterostructures as high-performance field-effect transistors," Nature 441, 489 (2006).

${ }^{2} \mathrm{~N}$. Singh et al., "Observation of metal-layer stress on Si nanowires in gateall-around high- $\kappa /$ metal-gate device structures," IEEE Electron Device Lett. 28(7), 558 (2007).

${ }^{3}$ C. Yang, Z. Zhong, and C. M. Lieber, "Encoding electronic properties by synthesis of axial modulation-doped silicon nanowires," Science 310, 1304 (2005)

${ }^{4} \mathrm{Q}$. Li et al., "Silicon nanowire on oxide/nitride/oxide for memory application," Nanotechnol. 18, 235204 (2007).

${ }^{5}$ Y. Hu, F. Kuemmeth, C. M. Lieber, and C. M. Marcus, "Hole spin relaxation in Ge-Si core-shell nanowire qubits," Nat. Nanotechnol. 7, 47 (2012).

${ }^{6}$ M. McAlpine, H. Ahmad, D. Wang, and K. J. Heath, "Highly ordered nanowire arrays on plastic substrates for ultrasensitive flexible chemical sensors," Nat. Mater. 6, 379 (2007).

${ }^{7}$ E. Stern et al., "Label-free immunodetection with CMOS-compatible semiconducting nanowires," Nature 445, 519 (2007).

${ }^{8} \mathrm{R}$. He and P. Yang, "Giant piezoresistance effect in silicon nanowires," Nat. Nanotechnol. 1, 42 (2006).

${ }^{9}$ X. L. Feng, R. He, P. Yang, and M. L. Roukes, "Very high frequency silicon nanowire electromechanical resonators," Nano Lett. 7(7), 1953 (2007). 
${ }^{10}$ A. I. Hochbaum et al., "Enhanced thermoelectric performance of rough silicon nanowires," Nature 451, 163 (2008).

${ }^{11}$ A. I. Boukai et al., "Silicon nanowires as efficient thermoelectric materials," Nature 451, 168 (2008).

${ }^{12} \mathrm{~B}$. Tian et al., "Coaxial silicon nanowires as solar cells and nanoelectronic power sources," Nature 449, 885 (2007).

${ }^{13} \mathrm{~V}$. Sivakov et al., "Silicon nanowire-based solar cells on glass: Synthesis, optical properties, and cell parameters," Nano Lett. 9(4), 1549 (2009).

${ }^{14}$ A. Zhang, H. Kim, J. Cheng, and Y.-H. Lo, "Ultrahigh responsivity visible and infrared detection using silicon nanowire phototransistors," Nano Lett. 10(6), 2117 (2010).

${ }^{15}$ Y. Ahn, J. Dunning, and J. Park, "Scanning photocurrent imaging and electronic band studies in silicon nanowire field effect transistors," Nano Lett. 5(7), 1367 (2005).

${ }^{16}$ C. Yang, C. J. Barrelet, F. Capasso, and C. M. Lieber, "Single p-type/ Intrinsic/n-type silicon nanowires as nanoscale avalanche photo detectors," Nano Lett. 6(12), 2929 (2006).

${ }^{17}$ O. Hayden, R. Agarwal, and C. M. Lieber, "Nanoscale avalanche photodiodes for highly sensitive and spatially resolved photon detection," Nat. Mater. 5, 352 (2006).

${ }^{18} \mathrm{O}$. Demichel et al., "Quantum confinement effects and strain-induced band-gap energy shifts in core-shell Si-SiO2 nanowires," Phys. Rev. B 83, 245443 (2011).

${ }^{19}$ R. Agarwal and C. M. Lieber, "Semiconductor nanowires: Optics and optoelectronics," Appl. Phys. A: Mater. Sci. Proc. 85, 209 (2006).

${ }^{20}$ Y. Huang, X. Duan, and C. M. Lieber, "Nanowires for integrated multicolor nanophotonics," Small 1(1), 142 (2005).

${ }^{21}$ M. Khorasaninejad and S. S. Saini, "Bend-waveguides on silicon nanowire optical waveguide (SNOW), " IEEE Photonics J. 3, 696-702 (2011).

${ }^{22}$ M. Khorasaninejad, N. Abedzadeh, J. Walia, S. Patchett, and S. S. Saini, "Color matrix refractive index sensors using coupled vertical silicon nanowire arrays," Nano Lett. 12(8), 4228 (2012).

${ }^{23} \mathrm{~L}$. Tsakalakos et al., "Strong broadband optical absorption in silicon nanowire films," J. Nanophotonics 1, 13552 (2007).

${ }^{24} \mathrm{~L}$. Hu and G. Chen, "Analysis of optical absorption in silicon nanowire arrays for photovoltaic applications," Nano Lett. 7(11), 3249 (2007).

${ }^{25} \mathrm{~J}$. Zhu et al., "Optical absorption enhancement in amorphous silicon nanowire and nanocone arrays," Nano Lett. 9(1), 279 (2009).

${ }^{26}$ M. M. Adachi, M. P. Anantram, and K. S. Karim, "Optical properties of crystalline-amorphous core-shell silicon nanowires," Nano Lett. 10(10), 4093 (2010).

${ }^{27}$ T.-M. Shih et al., "Supercollimation in photonic crystals composed of silicon rods," Appl. Phys. Lett. 93, 131111 (2008).

${ }^{28}$ A. Zhang, S. You, C. Soci, D. Wang, and Y.-H. Lo, in Planar and Vertical Si Nanowire Photo Detectors, Lasers and Electro-Optics, and Conference on Quantum Electronics and Laser Science, CLEO/QELS, San Jose, California, USA (2008)

${ }^{29}$ J. Ramanujam, D. Shiri, and A. Verma, "Silicon Nanowire Growth and Properties: A Review,” Mater. Express 1(2), 105-126 (2011).

${ }^{30}$ Y. Ishikawa et al., "Strain-induced band gap shrinkage in Ge grown on $\mathrm{Si}$ substrate," Appl. Phys. Lett. 82, 2044 (2003).

${ }^{31}$ T. Krishnamohan et al., "High performance, uniaxially-strained, silicon and germanium, double-gate p-MOSFETs," Microelectron. Eng. 84(9-10), 2063-2066 (2007).

${ }^{32}$ X. Sun, J. Liu, L. C. Kimerling, and J. Michel, "Room-temperature direct bandgap electroluminesence from Ge-on-Si light-emitting diodes," Opt. Lett. 34(8), 1198 (2009).

${ }^{33} \mathrm{X}$. Sun et al., "Electrically pumped hybrid evanescent Si/InGaAsP lasers," Opt. Lett. 34(9), 1345 (2009).

${ }^{34}$ D. Shiri, see http://hdl.handle.net/10012/7097 for Electronic and Optical Properties of Silicon Nanowires: Theory and Modeling, UWSpace, 2012.

${ }^{35}$ R. A. Soref and L. Friedman, "Direct-gap Ge/GeSn/Si and GeSn/Ge/Si heterostructures," Superlattices Microstruct. 14(2-3), 189 (1993).

${ }^{36}$ J. Menéndez and J. Kouvetakis, "Type-I Ge/ $\mathrm{Ge}_{1-\mathrm{x}-\mathrm{y}} \mathrm{Si}_{\mathrm{x}} \mathrm{Sn}_{\mathrm{y}}$ strained-layer heterostructures with a direct Ge bandgap," Appl. Phys. Lett. 85, 1175 (2004).

${ }^{37}$ D. C. Dillen, F. Wen, K. Kim, and E. Tutuc, "Coherently Strained $\mathrm{Si}-\mathrm{Si}_{\mathrm{x}} \mathrm{Ge}_{1-\mathrm{x}}$ Core-Shell Nanowire Heterostructures," Nano Lett. 16(1), 392 (2016).

${ }^{38} \mathrm{~K}$. E. Moselund et al., "Bended gate-all-around nanowire mosfet: A device with enhanced carrier mobility due to oxidation-induced stress," in IEDM Digest of Technical Papers (IEDM, 2007).
${ }^{39}$ N. Fukata et al., "Phonon confinement and self-limiting oxidation effect of silicon nanowires synthesized by laser ablation," J. Appl. Phys. 100, 024311 (2006).

${ }^{40} \mathrm{~A}$. Seike et al., "Strain-induced transconductance enhancement by pattern dependent oxidation in silicon nanowire field-effect transistors," Appl. Phys. Lett. 91, 202117 (2007).

${ }^{41}$ V. Pott et al., "Fabrication and characterization of gate-all-around silicon nanowires on bulk silicon," IEEE Trans. Nanotechnol. 7(6), 733 (2008).

${ }^{42} \mathrm{G}$. Audoit et al., "Strain induced photoluminescence from silicon and germanium nanowire arrays," J. Mater. Chem. 15, 4809 (2005).

${ }^{43}$ R. S. Jacobsen et al., "Strained silicon as a new electro-optic material," Nature 441, 199 (2006).

${ }^{44}$ M. Cazzanelli et al., "Second-harmonic generation in silicon waveguides strained by silicon nitride," Nat. Mater 11, 148 (2011).

${ }^{45}$ D. Shiri, A. Verma, C. R. Selvakumar, and M. P. Anantram, "Reversible modulation of spontaneous emission by strain in silicon nanowires," Sci. Rep. 2, 461 (2012).

${ }^{46}$ D. Shiri, Y. Kong, A. K. Buin, and M. P. Anantram, "Electromechanical response of silicon nanowires: Bandgap and effective mass," in Proceedings of International Semiconductor Device Research Symposium, College Park, MD, USA, 12-14 December 2007 (IEEE, Piscataway, NJ, USA, 2007), pp. 1-2.

${ }^{47}$ D. Shiri, Y. Kong, A. K. Buin, and M. P. Anantram, "Strain induced change of bandgap and effective mass in silicon nanowires," Appl. Phys. Lett. 93, 073114 (2008).

${ }^{48}$ Y. Kong, D. Shiri, and A. K. Buin, "First-principle investigation of strain effects on the electronic properties of germanium nanowires," Phys. Status Solidi RRL 3(9), 281 (2009).

${ }^{49}$ T. Maegawa, T. Hara, T. Yamauchi, H. Tsuchiya, and M. Ogawa, "Strain effects on electronic band structures in nano-scaled silicon: From bulk to nanowire," IEEE Trans. Electron Devices 56, 553 (2009).

${ }^{50} \mathrm{Z}$. Wu, J. B. Neaton, and C. Grossman, "Charge separation via strain in silicon nanowires," Nano Lett. 9(6), 2418 (2009).

${ }^{51}$ K.-H. Hong, J. Kim, S.-H. Lee, and J. K. Shin, "Strain-driven electronic band structure modulation of Si nanowires," Nano Lett. 8(5), 1335 (2008).

${ }^{52} \mathrm{P}$. W. Leu, A. Svizhenko, and K. Cho, "Ab initio calculations of the mechanical and electronic properties of strained Si nanowires," Phys. Rev. B 77, 235305 (2008).

${ }^{53}$ R. N. Sajjad and K. Alam, "Electronic properties of a strained $\langle 100\rangle$ silicon nanowire,” J. Appl. Phys. 105, 044307 (2009).

${ }^{54}$ E. M. Grumstrup, M. M. Gabriel, C. W. Pinion, J. K. Parker, J. F. Cahoon, and J. M. Papanikolas, "Reversible strain-induced electron-hole recombination in silicon nanowires observed with femto second pump-probe microscopy," Nano Lett. 14(11), 6287-6292 (2014).

${ }^{55}$ J.-M. Jancu, R. Scholz, F. Beltram, and F. Bassani, "Empirical spds* tightbinding calculation for cubic semiconductors: General method and material parameters," Phys. Rev. B 57, 6493 (1998).

${ }^{56}$ S. S. Walavalkar, C. E. Hofmann, A. P. Homyk, M. D. Henry, H. A. Atwater, and A. Scherer, "Tunable visible and near-IR emission from Sub$10 \mathrm{~nm}$ etched single-crystal Si nanopillars," Nano Lett. 10(11), 4423 (2010).

${ }^{57}$ J. M. Soler et al., "The SIESTA method for ab initio order-N materials simulation,” J. Phys.: Condens. Matter. 14, 2745 (2002).

${ }^{58} \mathrm{~A}$. Anselm, Introduction to Semiconductor Theory (Mir Publishers, Moscow, 1981).

${ }^{59}$ J. Noffsinger, E. Kioupakis, C. G. Van de Walle, S. G. Louie, and M. L. Cohen, "Phonon assisted optical absorption in silicon from first principles," Phys. Rev. Lett. 108, 167402 (2012).

${ }^{60}$ D. Shiri, A. Verma, and M. M. Khader, "Photoconductive response of strained silicon nanowires: A Monte Carlo study,” J. Appl. Phys. 115, 133708 (2014).

${ }^{61}$ J. P. Perdew and Y. Wang, "Accurate and simple analytic representation of the electron-gas correlation energy," Phys. Rev. B 45, 13244 (1992).

${ }^{62} \mathrm{C}$. Delerue and M. Lannoo, Nanostructures Theory and Modeling (Springer-Verlag, Berlin, Heidelberg, 2004).

${ }^{63}$ D. D. D. Ma, C. S. Lee, F. C. K. Au, S. Y. Tong, and S. T. Lee, "Smalldiameter silicon nanowire surfaces," Science 299, 1874 (2003).

${ }^{64} \mathrm{P}$. Nozières and D. Pines, "Electron interaction in solids; characteristic energy loss spectrum," Phys. Rev. 113, 1254 (1959).

${ }^{65}$ E. Eherenreich and M. H. Cohen, "Self-consistent field approach to the many-electron problem," Phys. Rev. 115, 786 (1959).

${ }^{66}$ S. L. Adler, "Quantum theory of the dielectric constant in real solids," Phys. Rev. 126, 413 (1962). 
${ }^{67}$ A. Marini, "Optical and electronic properties of copper and silver: From density functional theory to many body effects," Ph.D. dissertation (University of Rome - Tor Vergata, 2001).

${ }^{68} \mathrm{~F}$. Trani, "Electronic and optical properties of silicon nanocrystals: A tight binding study," Ph.D. dissertation (Università di Napoli "Federico II," 2004).

${ }^{69}$ M. R. Black, Y.-M. Lin, S. B. Cronin, O. Rabin, and M. S. Dresselhaus, "Infrared absorption in bismuth nanowires resulting from quantum confinement," Phys. Rev. B 65, 195417 (2002).

${ }^{70}$ G. D. Sanders and Y.-C. Chang, "Theory of optical properties of quantum wires in porous silicon," Phys. Rev. B 45, 9202 (1992).

${ }^{71} \mathrm{H}$. Yoshioka, "Fundamental study on si nanowires for advanced MOSFETs and light-emitting devices," Ph.D. dissertation (Kyoto University, 2010)

${ }^{72}$ P. Vogl, H. P. Hjalmarson, and J. D. Dow, “A semi-empirical tight binding theory of the electronic structure of semiconductors," J. Phys. Chem. Solids 44(5), 365-378 (1983).

${ }^{73}$ L. C. Lew Yan Voon and L. R. Ram-Mohan, "Tight-binding representation of the optical matrix elements: Theory and applications," Phys. Rev. B 47, 15500 (1993).

${ }^{74}$ Y.-C. Chang and D. E. Aspnes, "Theory of dielectric-function anisotropies of (001) GaAs $(2 \times 1)$ surfaces," Phys. Rev. B 41, 12002 (1990).

${ }^{75}$ N. A. Hill and K. B. Whaley, "Two-particle calculation of excitonic effects in semiconductor nanocrystals," Chem. Phys. 210, 117 (1996).

${ }^{76}$ T. B. Boykin and P. Vogl, "Dielectric response of molecules in empirical tight-binding theory," Phys. Rev. B 65, 035202 (2001).

${ }^{77}$ J. C. Slater, “Atomic shielding constants,” Phys. Rev. 36, 57 (1930).

${ }^{78}$ S. Lee, J. Kim, L. Jönsson, J. W. Wilkins, G. W. Bryant, and G. Klimeck, "Many-body levels of optically excited and multiply charged InAs nanocrystals modeled by semi empirical tight binding," Phys. Rev. B 66, 235307 (2002).

${ }^{79}$ See http://integrals.wolfram.com for Online integrator by Wolfram Alpha.

${ }^{80} \mathrm{~W}$. Harrison, Electronic Structure and the Properties of Solids-The Physics of the Chemical Bond (Dover Publications, Inc., New York, 1989).

${ }^{81}$ E. Rosencher and B. Vinter, Optoelectronics (Cambridge University Press, Cambridge, 2002).

${ }^{82}$ F. Szmulowicz and J. K. Wendeln, "Tetrahedral subdivision of the irreducible wedge for Brillouin zones of cubic lattices," Phys. Rev. B 25, 2428 (1982).

${ }^{83}$ S. Datta, Quantum Transport: Atom to Transistor (Cambridge University Press, Cambridge, 2005), Chap. 5.

${ }^{84}$ V. Lucarini, J. J. Saarinen, K.-E. Peiponen, and E. M. Vartiainen, Kramers-Krönig Relations in Optical Materials Research (SpringerVerlag, Berlin, Heidelberg, 2005).

${ }^{85}$ M. A. Green and M. J. Keevers, "Optical properties of intrinsic silicon at 300K," Prog. Photovoltaics 3(3), 189-192 (1995).

${ }^{86}$ Semiconductor-Basic Data, 2nd ed., edited by O. Madelung (SpringerVerlag, Berlin, Heidelberg, 1996).

${ }^{87}$ M. S. Hybertsen, "Absorption and emission of light in nanoscale silicon structures,” Phys. Rev. Lett. 72, 1514 (1994).

${ }^{88}$ Y. H. Ahn and J. Park, "Efficient visible light detection using individual germanium nanowire field effect transistors," Appl. Phys. Lett. 91, 162102 (2007).

${ }^{89}$ J. Wang, M. S. Gudiksen, X. Duan, Y. Cui, and C. M. Lieber, "Highly polarized photoluminescence and photo detection from single indium phosphide nanowires," Science 293, 1455 (2001). 